September 25, 2017

\title{
CARATHÈODORY'S METRICS ON TEICHMÜLLER SPACES AND L-SHAPED PILLOWCASES
}

\author{
VLADIMIR MARKOVIC
}

\begin{abstract}
One of the most important results in Teichmüller theory is the theorem of Royden which says that the Teichmüller and Kobayashi metrics agree on the Teichmüller space of a given closed Riemann surface. The problem that remained open is whether the Carathèodory metric agrees with the Teichmüller metric as well. In this paper we prove that these two metrics disagree on each $\mathcal{T}_{g}$, the Teichmüller space of a closed surface of genus $g \geq 2$. The main step is to establish a criterion to decide when the Teichmüller and Carathéodory metrics agree on the Teichmüller disc corresponding to a rational Jenkins-Strebel differential $\varphi$. First we construct a holomorphic embedding $\mathcal{E}: \mathbb{H}^{k} \rightarrow \mathcal{T}_{g, n}$ corresponding to $\varphi$. The criterion says that the two metrics agree on this disc if and only if a certain function $\Phi: \mathcal{E}\left(\mathbb{H}^{k}\right) \rightarrow \mathbb{H}$ can be extended to a holomorphic function $\boldsymbol{\Phi}: \mathcal{T}_{g, n} \rightarrow \mathbb{H}$. We then show by explicit computation that this is not the case for quadratic differentials arising from $L$-shaped pillowcases.
\end{abstract}

\section{INTRODUCTION}

1.1. Carathèodory vs. Kobayashi. A Schwarz-Pick system is a functor assigning to each complex manifold $X$ a pseudometric $d^{X}$ satisfying the following conditions:

(1) The metric assigned to the upper half-plane $\mathbb{H}=\{z: \operatorname{Im}(z)>0\}$ is the metric of constant curvature equal to -4 .

(2) Any holomorphic map $f: X \rightarrow Y$ between complex manifolds is nonexpanding:

$$
d^{Y}(f(p), f(q)) \leq d^{X}(p, q)
$$

for all $p, q \in X$.

Remark. We choose the metric on $\mathbb{H}$ to have constant curvature -4 (as opposed to -1 ) because this is more convenient in the context of Teichmüller theory as it is well known. Also, when the complex manifold $X$ is understood we simplify the notation by letting $d=d^{X}$.

The largest Schwarz-Pick pseudo-metric on $X$ is called the Kobayashi pseudometric $d_{K}$. On the other hand, one defines the Carathèodory pseudo-metric $d_{C}$ as

$$
d_{C}(p, q)=\sup \left\{d_{\mathbb{H}}(f(p), f(q)): f: X \rightarrow \mathbb{H}, \quad f \text { is holomorphic }\right\} .
$$

When either $d_{K}$ or $d_{C}$ happens to be a metric we refer to them as the Kobayashi and Carathèodory metrics respectively (see for example [7]).

2000 Mathematics Subject Classification. Primary $20 \mathrm{H} 10$.

This work was supported by the Simons Investigator Award 409745 from the Simons Foundation, by the Institute for Advanced Study, by the NSF grant number DMS-1201463, and by the Isaac Newton Institute in Cambridge 
The Schwarz lemma implies $d_{C}(p, q) \leq d_{K}(p, q)$ for $p, q \in X$. The question on which complex manifolds $X$ these two metrics agree received a good deal of attention. On the unit disc, and moreover on every poly-disc in $\mathbb{C}^{m}$, these two metrics agree. But examples of complex manifolds where these metrics agree are seldom.

In [16] Lempert showed that the Kobayashi and Carathèodory distances agree on convex domains. For quite a while these were the only known examples of domains where these metrics agree. In [1] and [3] it was established by AglerYoung and Costara, that the symmetrized bi-disc has this property while it is not bi-holomorphic to any convex domain.

In this paper we prove that these two metrics disagree on Teichmüller spaces of closed surfaces.

1.2. Carathoedory vs. Teichmüller. By $\mathcal{T}_{g, n}$ we denote the Teichmüller space of a genus $g$ surface with $n$ marked points and by $d_{\mathcal{T}}$ its Teichmüller metric. The space $\mathcal{T}_{g, n}$ is a contractible complex manifold of dimension $3 g-3+n$ which is bi-holomorphic to a domain in $\mathbb{C}^{3 g-3+n}$. As we already mentioned, Royden [19] showed that the Teichmüller metric agrees with the Kobayashi metric on $\mathcal{T}_{g}$. This result was extended to every $\mathcal{T}_{g, n}$ by Earle and Kra [6], and to all other Teichmüller spaces by Gardiner [9]. Therefore by the Schwarz lemma the inequality

$$
d_{C} \leq d_{\mathcal{T}}
$$

holds on every $\mathcal{T}_{g, n}$.

The Carathèodory metric $d_{C}$ has been studied on Teichmüller spaces. Earle showed [5] that $d_{C}$ is a complete metric on $\mathcal{T}_{g, n}$ and proportional with $d_{K}=d_{\mathcal{T}}$. As for the equality $d_{C}=d_{\mathcal{T}}$, it was established by Kra [15] (and later rediscovered by McMullen [18]) that these two metrics agree on Abelian Teichmüller discs in $\mathcal{T}_{g}$, and more generally on Teichmüller discs in $\mathcal{T}_{g, n}$ that are generated by holomorphic quadratic differentials whose zeroes are of even order. This beautiful theorem (proved using the period matrix map from $\mathcal{T}_{g}$ into the Siegel upper-half space) suggests that there is hope for establishing the equality on all Teichmüller discs and therefore on the entire Teichmüller space. However this is not the case.

Theorem 1.1. $d_{C} \neq d_{\mathcal{T}}$ on $\mathcal{T}_{0,5}$.

As a corollary of this theorem we prove the following.

Theorem 1.2. $d_{C} \neq d_{\mathcal{T}}$ on $\mathcal{T}_{g}$ for every $g \geq 2$.

Proof. The following argument is a variation of the argument used by Kra in Section 4 in [15]. The next claim is a known fact but we were not able to locate the exact reference so we provide a proof in the first appendix below.

Proposition 1.1. For each $g \geq 2$ there is a holomorphic and isometric embedding from $\mathcal{T}_{0,5}$ into $\mathcal{T}_{g}$ (isometric with respect to the Teichmüller metric).

Denote by $I: \mathcal{T}_{0,5} \rightarrow \mathcal{T}_{g}$ the corresponding embedding, that is $I$ is a holomorphic and an isometry onto its image. For every $p, q \in \mathcal{T}_{0,5}$ we have $d_{\mathcal{T}}(p, q)=$ $d_{\mathcal{T}}(I(p), I(q))$.

On the other hand, it follows from (1) that $d_{C}(I(p), I(q)) \leq d_{C}(p, q)$ (here we use the fact that $I$ is holomorphic). By Theorem 1.1 there exists $p, q \in \mathcal{T}(0,5)$ such 
that $d_{C}(p, q) \neq d_{\mathcal{T}}(p, q)$ and using $(2)$ we derive the strict inequality $d_{C}(p, q)<$ $d_{\mathcal{T}}(p, q)$. Thus $d_{C}(I(p), I(q)) \leq d_{C}(p, q)<d_{\mathcal{T}}(p, q)=d_{\mathcal{T}}(I(p), I(q))$ and we see that $d_{C}(I(p), I(q))<d_{\mathcal{T}}(I(p), I(q))$ which completes the proof.

1.3. The outline: Part 1. The rest of the paper is aimed at proving Theorem 1.1. There are two parts to it. We briefly describe each of them.

Let $S$ denote marked Riemann surface and $\varphi$ a holomorphic quadratic differential on $S$. By $\tau^{\varphi}: \mathbb{H} \rightarrow \mathcal{T}_{g, n}$ we denote the Teichmüller disc corresponding to $\varphi$. The Teichmüller and Carathèodory metrics agree on the disc $\tau^{\varphi}$ if and only if there exists a holomorphic map

$$
\Phi: \mathcal{T}_{g, n} \rightarrow \mathbb{H},
$$

such that $\left(\boldsymbol{\Phi} \circ \tau^{\varphi}\right)(\lambda)=\lambda$ for $\lambda \in \mathbb{H}$.

We assume that $\varphi$ is a rational Jenkins-Strebel differential which means that the moduli of the annuli $\Pi_{j}$ (swept out by closed horizontal trajectories of $\varphi$ ) have rational ratios. Then for a certain $t>0$ the translation $A_{t} \in \operatorname{Aut}(\mathbb{H})$, given by $A_{t}(\lambda)=\lambda+t$, belongs to the stabilizer of the disc $\tau^{\varphi}$. Moreover, $T=\tau_{*}^{\varphi}\left(A_{t}\right) \in$ $\operatorname{Mod}_{g, n}$ is a certain Dehn twist about curves homotopic to the annuli $\Pi_{j}$. Our first result, proved in Section 3, states that after averaging we may assume that the holomorphic function (3) is equivariant with respect to $T$ and $A_{t}$, that is

$$
\boldsymbol{\Phi} \circ T=A_{t} \circ \mathbf{\Phi} .
$$

We then show that such an invariant function $\boldsymbol{\Phi}$ has uniquely determined values on a certain subset of $\mathcal{T}_{g, n}$. Namely, in Section 4 we define a mapping $\mathcal{E}: \mathbb{H}^{k} \rightarrow \mathcal{T}_{g, n}$ where $\mathbb{H}^{k}$ is the poly-plane and $k$ the number of $\Pi_{j}$ 's (to properly define $\mathcal{E}$ we must assume that the annuli $\Pi_{j}$ are non-degenerate). The new marked Riemann surface $\mathcal{E}(\lambda)=S(\lambda), \lambda \in \mathbb{H}^{k}$, is obtained from $S$ by varying the heights and twists of the annuli $\Pi_{j}$ while the circumferences of $\Pi_{j}$ remain fixed. The map $\mathcal{E}$ is holomorphic but it only parametrizes a small part of $\mathcal{T}_{g, n}$ (however, if $k=3 g-3+n$ then $\mathcal{E}\left(\mathbb{H}^{k}\right)$ has a non-empty interior in $\left.\mathcal{T}_{g, n}\right)$.

In Section 5.2 we show that the restriction of $\boldsymbol{\Phi}$ to $\mathcal{E}\left(\mathbb{H}^{k}\right)$ is given by

$$
(\boldsymbol{\Phi} \circ \mathcal{E})\left(\lambda_{1}, \ldots, \lambda_{k}\right)=\alpha_{1} \lambda_{1}+\cdots+\alpha_{k} \lambda_{k},
$$

for some constants $\alpha_{j}>0$. We only prove this when $3 g-3+n=2$ for reasons of clarity and since in this paper we only require this case. But we expect this to hold in general.

Remark. If one wants to compute the Carathéodory distance on any complex manifold $X$ then at some point one has to prove a statement about all holomorphic functions $X \rightarrow \mathbb{H}$. The claim (4) is the corresponding statement in our case.

We can reformulate this result as the criterion for when the Carathéodory and Teichmüller metrics agree on $\tau^{\varphi}$. The criterion says that the two metrics agree on this disc if and only if the function $\boldsymbol{\Phi}: \mathcal{E}\left(\mathbb{H}^{k}\right) \rightarrow \mathbb{H}$ given by the formula (4) can be extended to a holomorphic function $\boldsymbol{\Phi}: \mathcal{T}_{g, n} \rightarrow \mathbb{H}$ (again, we prove this only when $3 g-3+n=2$ ). The proof of this criterion relies on the following rigidity result 
for equivariant holomorphic functions. Let $f: \mathbb{H}^{2} \rightarrow \mathbb{H}$ be a holomorphic function whose values on the diagonal in $\mathbb{H}^{2}$ are given by

$$
f(\eta, \eta)=\eta, \text { for every } \eta \in \mathbb{H},
$$

and which is translation invariant

$$
f(z+c, w+c)=f(z, w)+c, \text { for every }(z, w) \in \mathbb{H}^{2},
$$

and for some $c>0$. Then

$$
f(z, w)=\alpha_{1} z+\alpha_{2} w .
$$

This theorem is at the heart of our argument. Its proof is based on the Schwarz lemma and it can be found in Section 6. This completes the first part of the paper.

While the above criterion is a substantial claim about extremal holomorphic functions on $\mathcal{T}_{g, n}$, it is by no means obvious that it implies that the Carathèodory and Teichmüller metrics disagree on Teichmüller spaces. For example, there are Jenkins-Strebel differentials that are Abelian (or with even order zeroes) and in this case we know that the Carathèodory and Teichmüller metrics agree on the disc $\tau^{\varphi}$. The corresponding holomorphic function $\mathbf{\Phi}: \mathcal{T}_{g, n} \rightarrow \mathbb{H}$, that was constructed using the period matrix, is equivariant in the sense of (3). Moreover, one can check that the restriction of $\boldsymbol{\Phi}$ to $\mathcal{E}\left(\mathbb{H}^{k}\right)$ is given by the formula (4).

1.4. The Outline: Part 2. In the second part of the paper, we apply the criterion to Teichmüller discs in $\mathcal{T}_{0,5}$ arising from $L$-shaped pillowcases. For $a, b>0$ and $0<q<1$ we let $L(a, b, q)$ denote the $L$-shaped polygon as in Figure 1 . The $L$ shaped pillowcase $S(a, b, q)$ is the double of the polygon $L(a, b, q)$ and is formally defined as a half-translation surface. The $(2,0)$ form $d z^{2}$ on $L(a, b, q)$ gives rise to the Jenkins-Strebel quadratic differential $\psi(a, b, q)$ on $S(a, b, q)$. (The surface $S(a, 0, q) \in \mathcal{T}_{0,5}$ is also well defined and it is the usual pillowcase surface with an extra marked point on one of its edges).

Assuming that the Carathèodory and Teichmüller metrics agree on $\mathcal{T}_{0,5}$, for each $q_{0} \in(0,1)$ we construct a holomorphic function $\boldsymbol{\Psi}: \mathcal{T}_{0,5} \rightarrow \mathbb{H}$, whose restriction to the locus $S\left(a, b, q_{0}\right)$ (we vary $a$ and $b$ but $q_{0}$ remains fixed) is given by

$$
\boldsymbol{\Psi}\left(S\left(a, b, q_{0}\right)\right)=\left(a+b q_{0}\right) \mathbf{i} .
$$

We show that such a map $\boldsymbol{\Psi}$ can not be holomorphic at points $S\left(a, 0, q_{0}\right) \in \mathcal{T}_{0,5}$. This is shown by considering the smooth path $S(t)=S\left(a, 0, q_{0}-t\right)$ in $\mathcal{T}_{0,5}$. We compute the first and second derivatives of $\boldsymbol{\Psi}(S(t))$ and verify that $\boldsymbol{\Psi}$ is not twice differentiable at $S(0)$.

Most of the work in this part of the paper is to compute the values $\boldsymbol{\Psi}(S(t))$. In order to compute $\boldsymbol{\Psi}\left(S\left(a, 0, q_{0}-t\right)\right)$ we need to find $a(t)$ and $b(t)$ such that the marked surfaces $S\left(a, 0, q_{0}-t\right)$ and $S\left(a(t), b(t), q_{0}\right)$ represent the same point in $\mathcal{T}_{0,5}$. We do this using the formula for Schwarz-Christoffel maps from $\mathbb{H}$ to $L$-shaped polygons $L(a, b, c)$.

This last few pages of the paper are little more technical although we only perform advanced high school mathematics. It would be important to find a way of generalizing this computation and applying the above criterion to Teichmüller discs corresponding to Jenkins-Strebel differentials in arbitrary $\mathcal{T}_{g, n}$. 


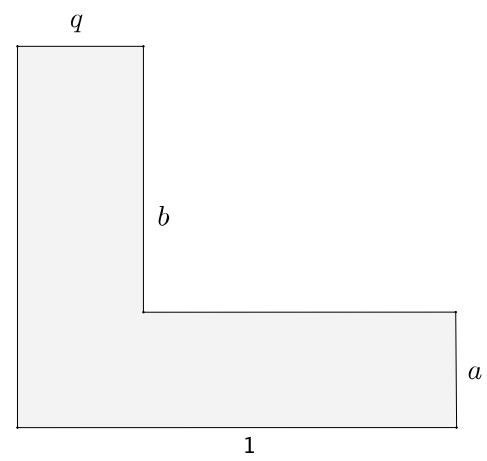

FigURE 1. $L$-shaped polygon $L(a, b, q)$

1.5. Acknowledgment. We make several remarks comparing different aspects of our work with the papers by McMullen [18] and Knese [14]. Although we actually do not use any of the results from [18] and [14], these papers have influenced the writing of this one.

We thank the referees for very useful suggestions that have improved the paper. Also, we thank F. Gradiner for sending us comments and the paper [10]. S. Antonakoudis has also reported making significant progress towards understanding Carathéodory's metric on Teichmüller spaces.

\section{TeIChMüLLER DISCS}

2.1. Teichmüller discs. Let $\Sigma_{g, n}$ denote an oriented smooth surface of genus $g \geq 2$ with $n$ marked points (once and for all we assume $3 g-3+n>0$ ). Recall that $\mathcal{T}_{g, n}$ is the space of marked Riemann surfaces $S$ of type $(g, n)$, each equipped with an orientation-preserving homeomorphism $\Sigma_{g, n} \rightarrow S$ up to isotopy.

By $\operatorname{Mod}_{g, n}$ we denote the corresponding Modular Group (also known as the Mapping Class Group) and by $\mathcal{M}_{g, n}=\mathcal{T}_{g, n} / \operatorname{Mod}_{g, n}$ the corresponding Moduli space. Let $\pi_{g, n}: \mathcal{T}_{g, n} \rightarrow \operatorname{Mod}_{g, n}$ denote the projection.

We let $\mathcal{Q D}(S)$ and $\mathcal{B D}(S)$ respectively denote the spaces of holomorphic quadratic differentials and Beltrami differentials on $S$. Also, let $\mathcal{B D}_{1}(S)$ denote the unit ball (in the $L^{\infty}$ norm) in $\mathcal{B D}(S)$. Each $\mu \in \mathcal{B D}_{1}(S)$ defines the point $[\mu] \in \mathcal{T}_{g, n}$ and the corresponding map $\mathcal{B D}_{1}(S) \rightarrow \mathcal{T}(S)$ is holomorphic.

A holomorphic quadratic differential $\varphi \in \mathcal{Q D}(S)$ induces a holomorphic map from the upper half plane $\mathbb{H}$ to $\mathcal{B D}_{1}(S)$ given by $\lambda \rightarrow\left(\frac{\mathbf{i}-\lambda}{\mathbf{i}+\lambda}\right) \frac{|\varphi|}{\varphi}$. In turn this defines the holomorphic embedding $\tau^{\varphi}: \mathbb{H} \rightarrow \mathcal{T}_{g, n}$ by letting

$$
\tau^{\varphi}(\lambda)=\left[\left(\frac{\mathbf{i}-\lambda}{\mathbf{i}+\lambda}\right) \frac{|\varphi|}{\varphi}\right] \in \mathcal{T}_{g, n} .
$$

Note that $\tau^{\varphi}(\mathbf{i})=S$. We refer to the map $\tau^{\varphi}$ as the Teichmüller disc. 
Let $S(\lambda)=\tau^{\varphi}(\lambda)$. The induced quasiconformal map $S \rightarrow S(\lambda)$, with the dilatation $\left(\frac{\mathbf{i}-\lambda}{\mathbf{i}+\lambda}\right) \frac{|\varphi|}{\varphi}$ is affine in local coordinates corresponding to $\varphi$ and the corresponding terminal quadratic differential $\varphi(\lambda) \in \mathcal{Q D}(S(\lambda))$. If $z$ is the local parameter on $S$ such that $\varphi=d z^{2}$ then the quasiconformal map is of the form

$$
z \rightarrow x+\lambda y
$$

where $z=x+y \mathbf{i}$.

\subsection{Stabilizing Teichmüller discs. We let}

$$
\operatorname{Stab}\left(\tau^{\varphi}\right)=\left\{A \in \operatorname{Aut}(\mathbb{H}): \pi_{g, n} \circ \tau^{\varphi} \circ A=\pi_{g, n} \circ \tau^{\varphi}\right\},
$$

denote the stabilizer of $\tau^{\varphi}$. There are the induced monomorphism

$$
\tau_{*}^{\varphi}: \operatorname{Stab}(\tau) \rightarrow \operatorname{Mod}_{g, n},
$$

and the induced quotient map $\mathbb{H} / \operatorname{Stab}\left(\tau^{\varphi}\right) \rightarrow \mathcal{M}_{g, n}$. When $\mathbb{H} / \operatorname{Stab}\left(\tau^{\varphi}\right)$ has finite hyperbolic area we refer to $\tau^{\varphi}$ as a Teichmüller curve.

Suppose $\varphi \in \mathcal{Q D}(S)$ is a J-S differential (J-S stand for Jenkins-Strebel), that is $\varphi$ induces a decomposition of $S$ into a finite number of annuli $\Pi_{j}, j=1, \ldots, k$, foliated by closed horizontal trajectories of $\varphi$. Let $\gamma_{1}, \ldots \gamma_{k}$ be a collection of disjoint simple closed curves on $S$ homotopic to $\Pi_{j}$ 's.

By $T_{\gamma_{j}} \in \operatorname{Mod}_{g, n}$ we denote the Dehn twist about $\gamma_{j}$. The following proposition is well known and elementary (see the analogous Lemma 9.7 in [18], but note that this lemma concerns the annuli swept out by closed vertical trajectories). Let $m_{j}$ denote the conformal modulus of $\Pi_{j}$. If $m_{j}$ 's have rational ratios we call $\varphi$ a rational J-S differential.

Proposition 2.1. Let $\varphi$ be a rational $J$-S differential and let

$$
t=\operatorname{lcm}\left\{m_{1}^{-1}, \ldots, m_{k}^{-1}\right\},
$$

where $\mathrm{lcm}$ stands for the lowest common multiple. Set $A_{t}(\lambda)=\lambda+t, \lambda \in \mathbb{H}$. Then $A_{t} \in \operatorname{Stab}\left(\tau^{\varphi}\right)$ and $\tau_{*}^{\varphi}\left(A_{t}\right)=T \in \operatorname{Mod}_{g, n}$ is the product of the (commuting) Dehn twists $T=T_{\gamma_{1}}^{n_{1}} \cdots T_{\gamma_{k}}^{n_{k}}$, where $n_{j}=m_{j}$ t.

\section{Carathéodory metric on Teichmüller discs}

3.1. Extremal discs for holomorphic functions on Teichmüller spaces. Fix $\varphi \in \mathcal{Q D}(S)$ and let $\boldsymbol{\Phi}: \mathcal{T}_{g, n} \rightarrow \mathbb{H}$ be a holomorphic map. We say that the Teichmüller disc $\tau^{\varphi}$ is an extremal disc for $\boldsymbol{\Phi}$ if $\left(\boldsymbol{\Phi} \circ \tau^{\varphi}\right) \in \operatorname{Aut}(\mathbb{H})$

By Royden's theorem each Teichmüller disc is an isometric embedding of $\mathbb{H}$ (with respect to its hyperbolic metric) into $\mathcal{T}_{g, n}$ (with respect to its Teichmüller metric). Thus, the Teichmüller and Carathèodory metrics agree on a Teichmüller disc $\tau^{\varphi}$ if an only if $\tau^{\varphi}$ is an extremal disc for some holomorphic map $\Phi: \mathcal{T}_{g, n} \rightarrow \mathbb{H}$.

We record the following elementary proposition.

Proposition 3.1. Fix $\varphi \in \mathcal{Q D}(S)$ and let $\boldsymbol{\Phi}: \mathcal{T}_{g, n} \rightarrow \mathbb{H}$ be any holomorphic map such that $\left(\boldsymbol{\Phi} \circ \tau^{\varphi}\right)(\lambda)=\lambda$, for every $\lambda \in \mathbb{H}$. The derivative of $\boldsymbol{\Phi}$ at $S(\lambda)=\tau^{\varphi}(\lambda)$ is given by

$$
d \boldsymbol{\Phi}(\nu)=\frac{-2 \mathbf{i} \operatorname{Im}(\lambda)}{\|\varphi(\lambda)\|_{1}} \int_{S(\lambda)} \nu \varphi(\lambda),
$$


where $\nu \in \mathcal{B D}(S(\lambda))$ represents a tangent vector to $\mathcal{T}_{g, n}$ at the point $S(\lambda)$ and $\|\varphi(\lambda)\|_{1}$ stands for the $L^{1}$-norm of $\varphi(\lambda) \in \mathcal{Q D}(S(\lambda))$.

Proof. Let $A: \mathbb{H} \rightarrow \mathbb{D}$ be the Möbius map given by

$$
A(z)=\frac{z-\lambda}{z-\bar{\lambda}}
$$

Note $A(\lambda)=0$. Set $\boldsymbol{\Psi}=A \circ \boldsymbol{\Phi}$. Then $\boldsymbol{\Psi}: \mathcal{T}_{g, n} \rightarrow \mathbb{D}$ is holomorphic and there exists a holomorphic quadratic differential $\psi$ on $S(\lambda)$ such that

$$
d \boldsymbol{\Psi}(\nu)=\int_{S(\lambda)} \nu \psi,
$$

for every $\nu \in \mathcal{B D}(S(\lambda))$. Moreover, since $\boldsymbol{\Psi}\left(\mathcal{T}_{g, n}\right) \subset \mathbb{D}$ and $\boldsymbol{\Psi}(S(\lambda))=0$, it follows from the Schwarz lemma and the Royden's theorem that $|d \boldsymbol{\Psi}(\nu)| \leq\|\nu\|_{\infty}$. This yields

$$
\|\psi\|_{1} \leq 1
$$

On the other hand, let $f: \mathbb{D} \rightarrow \mathcal{T}_{g, n}$ be given by $f=\tau^{\varphi} \circ A^{-1}$. From the definition of the Teichmüller disc $\tau^{\varphi}$ and the choice of the Möbius map $A$, it follows that $f$ is the Teichmüller disc given by

$$
f(\eta)=\left[\eta \frac{|\varphi(\lambda)|}{\varphi(\lambda)}\right] \in \mathcal{T}_{g, n} .
$$

Moreover, $(\boldsymbol{\Psi} \circ f)(\eta)=\eta$ for every $\eta \in \mathbb{D}$. Thus

$$
d \Psi\left(\frac{d f}{d \eta}\right)=1
$$

Since $\frac{d f}{d \eta}=\frac{|\varphi(\lambda)|}{\varphi(\lambda)}$, after combining (10) with (9) we get $\psi=\frac{\varphi(\lambda)}{\|\varphi(\lambda)\|_{1}}$, that is

$$
d \Psi(\nu)=\frac{1}{\|\varphi(\lambda)\|_{1}} \int_{S(\lambda)} \nu \varphi(\lambda),
$$

for every $\nu \in \mathcal{B D}(S(\lambda))$. From $\boldsymbol{\Psi}=A \circ \boldsymbol{\Phi}$ we get

$$
d \boldsymbol{\Phi}(\nu)=\frac{1}{A^{\prime}(\lambda)} d \mathbf{\Psi}(\nu) .
$$

Replacing

$$
A^{\prime}(\lambda)=\frac{1}{-2 \mathbf{i} \operatorname{Im}(\lambda)}
$$

in the previous identity yields the proof. 
3.2. Equivariant holomorphic functions. Assume $\varphi$ to be a rational J-S differential and $\widehat{\boldsymbol{\Phi}}: \mathcal{T}_{g, n} \rightarrow \mathbb{H}$ to be a holomorphic function such that the Teichmüller disc $\tau^{\varphi}$ is extremal for $\widehat{\boldsymbol{\Phi}}$. We want to average $\widehat{\boldsymbol{\Phi}}$ (in a suitable sense) over the cyclic group generated by the twist $T \in \operatorname{Mod}_{g, n}$ from Proposition 2.1 and show that one can replace $\widehat{\boldsymbol{\Phi}}$ with an equivariant holomorphic function $\boldsymbol{\Phi}$ that also has the property that $\tau^{\varphi}$ is an extremal disc for $\boldsymbol{\Phi}$.

Lemma 3.1. Suppose $\varphi$ is a rational J-S differential on a marked Riemann surface $S$. If $\tau^{\varphi}$ is extremal for a holomorphic function $\widehat{\mathbf{\Phi}}: \mathcal{T}_{g, n} \rightarrow \mathbb{H}$ then there exists a holomorphic function $\mathbf{\Phi}: \mathcal{T}_{g, n} \rightarrow \mathbb{H}$ with the following properties

(1) $\left(\Phi \circ \tau^{\varphi}\right)(\lambda)=\lambda$, for every $\lambda \in \mathbb{H}$,

(2) $(\boldsymbol{\Phi} \circ T)(\tau)=\boldsymbol{\Phi}(\tau)+t$, for every $\tau \in \mathcal{T}_{g, n}$, where $T=\tau_{*}^{\varphi}\left(A_{t}\right)$ is the Dehn twist from Proposition 2.1.

Proof. After post-composing $\widehat{\boldsymbol{\Phi}}$ by an automorphism of $\mathbb{H}$ if necessary, we may assume $\left(\widehat{\boldsymbol{\Phi}} \circ \tau^{\varphi}\right)(\lambda)=\lambda$ for every $\lambda \in \mathbb{H}$. Let

$$
\widehat{\boldsymbol{\Phi}}_{m}(\tau)=\frac{1}{m} \sum_{k=0}^{m-1}\left(\widehat{\boldsymbol{\Phi}}\left(T^{k}(\tau)\right)-k t\right), \text { for every } \quad \tau \in \mathcal{T}_{g, n} .
$$

Clearly $\widehat{\boldsymbol{\Phi}}_{m}: \mathcal{T}_{g, n} \rightarrow \mathbb{H}$. Since $T=\tau_{*}^{\varphi}\left(A_{t}\right)$ and $A_{t} \in \operatorname{Stab}\left(\tau^{\varphi}\right)$ we conclude $\left(\widehat{\mathbf{\Phi}}_{m} \circ \tau^{\varphi}\right)(\lambda)=\lambda$, for every $\lambda \in \mathbb{H}$.

The sequence $\widehat{\boldsymbol{\Phi}}_{m}: \mathcal{T}_{g, n} \rightarrow \mathbb{H}$ is a normal family (since $\mathbb{H}$ is conformally the same as the unit disc). Thus the sequence $\widehat{\boldsymbol{\Phi}}_{m}$ has an accumulation point. The restriction of $\widehat{\boldsymbol{\Phi}}_{m}$ to the Teichmüller disc $\tau^{\varphi}(\mathbb{H})$ does not change with $m$, so every accumulation point $\mathbf{\Phi}: \mathcal{T}_{g, n} \rightarrow \mathbb{H}$ of this sequence is a non constant holomorphic map.

We want to show that every such accumulation point $\boldsymbol{\Phi}$ is an equivariant function. We compute

$$
\widehat{\mathbf{\Phi}}_{m}(T(\tau))-\widehat{\boldsymbol{\Phi}}_{m}(\tau)=\frac{\widehat{\boldsymbol{\Phi}}\left(T^{m}(\tau)\right)}{m}-\frac{\widehat{\mathbf{\Phi}}(\tau)}{m} .
$$

Clearly $\frac{\widehat{\boldsymbol{\Phi}}(\tau)}{m} \rightarrow 0$ when $m \rightarrow \infty$ and for a fixed $\tau$. Next we show

$$
\frac{\widehat{\boldsymbol{\Phi}}\left(T^{m}(\tau)\right)}{m} \rightarrow t
$$

This can be seen from the Schwarz lemma as follows.

Let $\tau_{0}=\tau^{\varphi}(\mathbf{i})$. Since $T^{m}$ is an isometry of $\mathcal{T}_{g, n}$ it follows that

$$
d_{\mathcal{T}}\left(T^{m}\left(\tau_{0}\right), T^{m}(\tau)\right)=d_{\mathcal{T}}\left(\tau_{0}, \tau\right)=C(\tau),
$$

for some constant $C(\tau)$ that only depends on $\tau$. From the Schwarz lemma we conclude

$$
d_{\mathbb{H}}\left(\widehat{\mathbf{\Phi}}\left(T^{m}\left(\tau_{0}\right)\right), \widehat{\mathbf{\Phi}}\left(T^{m}(\tau)\right)\right) \leq C(\tau) .
$$

But $\widehat{\mathbf{\Phi}}\left(T^{m}\left(\tau_{0}\right)\right)=\mathbf{i}+m t$. Combining this with the previous inequality yields the estimate

$$
\left|\widehat{\mathbf{\Phi}}\left(T^{m}(\tau)\right)-(\mathbf{i}+m t)\right| \leq C_{1}(\tau),
$$


where $C_{1}(\tau)$ is some other constant depending only on $\tau$. Dividing both sides by $m$, yields $\frac{\widehat{\Phi}\left(T^{m}(\tau)\right)}{m} \rightarrow t$, as promised.

Replacing this into (12) yields the equality

$$
\widehat{\boldsymbol{\Phi}}_{m}(T(\tau))-\widehat{\boldsymbol{\Phi}}_{m}(\tau)=t+o(1),
$$

where $o(1) \rightarrow 0$ when $m \rightarrow \infty$. This proves that any accumulation point $\boldsymbol{\Phi}$ of the sequence $\widehat{\boldsymbol{\Phi}}_{m}$ satisfies the equivariance condition

$$
\mathbf{\Phi} \circ T=A_{t} \circ \mathbf{\Phi}
$$

and the proof is complete.

\section{Mapping the POly-Plane $\mathbb{H}^{k}$ TO $\mathcal{T}_{g, n}$}

4.1. Definitions. Fix a marked Riemann surface $S \in \mathcal{T}_{g, n}$. Select a rational J-S quadratic differential $\varphi \in \mathcal{Q D}(S)$ and let $h_{1}, \ldots, h_{k}$ denote the heights (with respect to the $|\varphi|$ singular metric) of the corresponding annuli $\Pi_{j}$. To define the poly-pane mapping below we must assume that each $h_{j}>0$ (that is $\Pi_{j}$ is non-degenerate).

We let $\mathbb{H}^{k}=\mathbb{H} \times \cdots \times \mathbb{H}$ denote the $k$-fold product of the upper half plane $\mathbb{H}$, and let $\lambda=\left(\lambda_{1}, \ldots, \lambda_{k}\right)$ denote the coordinates on $\mathbb{H}^{k}$. Define $\mathcal{F}: \mathbb{H}^{k} \rightarrow \mathcal{B} \mathcal{D}_{1}(S)$ by letting

$$
\mathcal{F}(\lambda)=\left(\frac{\mathbf{i}-\lambda_{j}}{\mathbf{i}+\lambda_{j}}\right) \frac{|\varphi|}{\varphi},
$$

on each $\Pi_{j}$. This yields the map $\mathcal{E}: \mathbb{H}^{k} \rightarrow \mathcal{T}_{g, n}$ by letting $\mathcal{E}(\lambda)=[\mathcal{F}(\lambda)]$. We say that $\mathcal{E}$ is the poly-plane mapping corresponding to $\varphi$.

The map $\mathcal{F}$ is clearly holomorphic and thus $\mathcal{E}$ is holomorphic as well. Observe that the restriction of $\mathcal{E}$ on the diagonal in $\mathbb{H}^{k}$ is the Teichmüller disc $\tau^{\varphi}$.

The new marked Riemann surface $\mathcal{E}(\lambda)=S(\lambda)$ comes equipped with the quadratic differential $\varphi(\lambda)$ which is the unique J-S differential in $\mathcal{Q D}(S(\lambda))$ which induces a decomposition of $S(\lambda)$ into annuli $\Pi_{j}(\lambda)$ (swept out by closed horizontal trajectories homotopic to $\gamma_{j}$ ) such that the height $h_{j}(\lambda)$ of $\Pi_{j}(\lambda)$ is given by $h_{j}(\lambda)=\operatorname{Im}\left(\lambda_{j}\right) h_{j}$. (By the Hubbard-Masur theorem [12] such $\varphi(\lambda)$ exists and is unique.)

The quasiconformal map with the Beltrami dilatation $\mathcal{F}(\lambda)$ is affine (with respect to the coordinates corresponding to $\varphi$ and $\varphi(\lambda)$ respectively) on each annulus. Moreover, for every $\lambda, \widehat{\lambda} \in \mathbb{H}^{k}$ the affine map between the annuli $\Pi_{j}(\lambda)$ and $\Pi_{j}(\hat{\lambda})$ is of the form (compare with (6))

$$
z \rightarrow x+\left(\frac{\widehat{\lambda}_{j}-\operatorname{Re}\left(\lambda_{j}\right)}{\operatorname{Im}\left(\lambda_{j}\right)}\right) y,
$$

where $z=x+\mathbf{i} y$ is the local coordinate on the surface $S(\lambda)$ in which the differential if of the form $\varphi(\lambda)=d z^{2}$.

Let $m_{j}$ denote the conformal modulus of the annulus $\Pi_{j}$. It is evident from (13) that the map $\mathcal{E}$ conjugates the translation $\lambda \rightarrow\left(\lambda+\left(0, . ., m_{j}^{-1}, \ldots, 0\right)\right)$ to the twist 
$T_{\gamma_{j}} \in \operatorname{Mod}_{g, n}$, that is

$$
\mathcal{E}\left(\lambda+\left(0, . ., m_{j}^{-1}, \ldots, 0\right)\right)=\left(T_{\gamma_{j}} \circ \mathcal{E}\right)(\lambda),
$$

for every $\lambda \in \mathbb{H}^{k}$. This yields the equality

$$
\mathcal{E}(\lambda+(t, \ldots, t))=(T \circ \mathcal{E})(\lambda),
$$

where $t$ is given by (7) and $T \in \operatorname{Mod}_{g, n}$ is the corresponding Dehn twist from Proposition 2.1 above.

Remark. We make a few observations and claims which we neither need nor prove.

- In [17] Liu constructs Fenchel-Nielsen type coordinates for $\mathcal{T}_{g, n}$. On each marked Riemann surface he finds the unique J-S quadratic differential with the corresponding annuli about $\gamma_{j}$ 's having the prescribed heights (this of course follows from the Hubbar-Masur theorem [12]). The coordinates are the circumferences of the annuli and the twist parameters (that determine how adjacent annuli are glued to each other). These coordinates should not be confused with our map $\mathcal{E}$. In our case the circumferences stay fixed and heights are the ones that vary.

- The map $\mathcal{E}$ is an embedding. This follows from the theorem of Jenkins [13] which states that there exists at most one Jenkins-Strebel differential such that the corresponding annuli have prescribed circumferences.

- Our "coordinates" are holomorphic but at the expense that they parametrize only a small part of $\mathcal{T}_{g, n}$.

- The map $\mathcal{E}$ is far from being proper.

\section{CRITERION FOR CARATHÉOdORY=TEICHMÜLLER}

In this section we assume that $\varphi$ is a J-S differential and that $\boldsymbol{\Phi}: \mathcal{T}_{g, n} \rightarrow \mathbb{H}$ is a holomorphic function satisfying the conditions (1) and (2) from Lemma 3.1. We establish the criterion for deciding when the two metrics agree on this Teichmüller disc.

5.1. Computing the derivatives of $\boldsymbol{\Phi}: \mathcal{T}_{g, n} \rightarrow \mathbb{H}$. Let $\gamma_{j}$ stand for simple closed curves on $S$ homotopic to the corresponding annuli $\Pi_{j}$ (which are swept out by closed horizontal trajectories of $\varphi$ ). Let $f=\boldsymbol{\Phi} \circ \mathcal{E}$, where $\mathcal{E}$ is the poly-plane map corresponding to $\varphi$. Then $f: \mathbb{H}^{k} \rightarrow \mathbb{H}$. Moreover, the values of $f$ on the diagonal in $\mathbb{H}^{k}$ are given by

$$
f(\eta, \eta, \ldots, \eta)=\eta
$$

for every $\eta \in \mathbb{H}$.

Let us compute the derivatives of $f$ along the diagonal. Let $\lambda=(\eta, \eta, \ldots, \eta)$. Fix $\eta$ and let $h \in \mathbb{C}$. The marked surface $S(\eta, \ldots,(\eta+h), \ldots, \eta)$ is a quasiconformal deformation of $S(\eta, \ldots, \eta)$. Moreover, from (13) we compute $S(\eta, \ldots,(\eta+h), \ldots, \eta)=$ $\left[h \mu_{j}+o(h)\right]$, where $\mu_{j} \in \mathcal{B D}(S(\lambda))$ is equal to zero outside the annulus $\Pi_{j}(\lambda)$ and on $\Pi_{j}(\lambda)$ is given by

$$
\mu_{j}=\frac{\mathbf{i}}{2 \operatorname{Im}(\eta)} \frac{|\varphi(\lambda)|}{\varphi(\lambda)} .
$$

Also, $o(h) \in \mathcal{B D}\left(\Pi_{j}(\lambda)\right)$ is such that $o(h) / h \rightarrow 0$ when $h \rightarrow 0$. 
According to the formula (8) from Proposition 3.1 the (complex) derivative of $\Phi$ at the point $S(\lambda)=\mathcal{E}(\lambda)$ is

$$
d \boldsymbol{\Phi}(\nu)=\frac{-2 \mathbf{i} \operatorname{Im}(\eta)}{\|\varphi(\lambda)\|_{1}} \int_{S(\lambda)} \nu \varphi(\lambda)
$$

where $\nu \in \mathcal{B D}(S(\lambda))$ represents a tangent vector to $\mathcal{T}_{g, n}$ at the point $S(\lambda)$.

After replacing $\nu=\mu_{j}$ we get

$$
\frac{\partial f}{\partial \lambda_{j}}(\lambda)=\frac{-2 \mathbf{i} \operatorname{Im}(\eta)}{\|\varphi(\lambda)\|_{1}} \int_{\Pi_{j}(\lambda)} \frac{\mathbf{i}}{2 \operatorname{Im}(\eta)}|\varphi(\lambda)|=\frac{1}{\|\varphi(\lambda)\|_{1}} \int_{\Pi_{j}(\lambda)}|\varphi(\lambda)| .
$$

We have just established the following proposition.

Proposition 5.1. For $\lambda=(\eta, \ldots, \eta)$ we have

$$
\frac{\partial f}{\partial \lambda_{j}}(\lambda)=\alpha_{j}
$$

where

$$
\alpha_{j}=\frac{1}{\|\varphi(\lambda)\|_{1}} \int_{\Pi_{j}(\lambda)}|\varphi(\lambda)|=\frac{1}{\|\varphi\|_{1}} \int_{\Pi_{j}}|\varphi|,
$$

and $\Pi_{j}, j=1, \ldots k$, are the corresponding annuli swept out by closed horizontal trajectories (note $\alpha_{1}+\cdots+\alpha_{k}=1$ ).

5.2. The Criterion. From (14) and from (2) in Lemma 3.1 it follows

$$
f(\lambda+(t, \ldots, t))=f(\lambda)+t, \quad \text { for every } \lambda \in \mathbb{H}^{k} .
$$

Proposition 5.1 yields

$$
\frac{\partial f}{\partial \lambda_{j}}(\lambda)=\alpha_{j}, \quad \text { for } \quad \lambda=(\eta, \ldots, \eta)
$$

where

$$
\alpha_{j}=\frac{1}{\|\varphi\|_{1}} \int_{\Pi_{j}(\lambda)}|\varphi|
$$

Below in Theorem 6.1 we prove that any holomorphic function $f: \mathbb{H}^{2} \rightarrow \mathbb{H}$ satisfying (15), (16), and (17), must be equal to

$$
f\left(\lambda_{1}, \lambda_{2}\right)=\alpha_{1} \lambda_{1}+\alpha_{2} \lambda_{2}, \quad \text { for every }\left(\lambda_{1}, \lambda_{2}\right) \in \mathbb{H}^{2} .
$$

Remark. Analogous result holds true for functions on the poly-plane $\mathbb{H}^{k}$ of arbitrary dimension. Here we prove it only in the case $k=2$ for the reasons of clarity and since we only apply this theorem in that case.

Now we prove the following corollary of Theorem 6.1.

Theorem 5.1. Suppose $3 g-3+n=2$. Let $\varphi$ be a rational J-S differential that induces $S$ to decompose into exactly two (non-degenerate) annuli swept out by closed horizontal trajectories of $\varphi$. Then any holomorphic function $\boldsymbol{\Phi}: \mathcal{T}_{g, n} \rightarrow \mathbb{H}$ satisfying the conditions (1) and (2) from Lemma 3.1 has the property that its restriction on the set $\mathcal{E}\left(\mathbb{H}^{2}\right)$ is given by the formula 
for every $\left(\lambda_{1}, \lambda_{2}\right) \in \mathbb{H}^{2}$.

$$
(\boldsymbol{\Phi} \circ \mathcal{E})\left(\lambda_{1}, \lambda_{2}\right)=\alpha_{1} \lambda_{1}+\alpha_{2} \lambda_{2}
$$

Proof. The function $f$ satisfies the assumptions of Theorem 6.1 and we verify the formula in the statement of this theorem.

We have now established the following criterion to decide when the Carathérode and Teichüller metrics agree on the Teichmülelr disc $\tau^{\varphi}$.

Theorem 5.2. Suppose $3 g-3+n=2$. Let $\varphi$ be a rational $J-S$ differential that induces $S$ to decompose into exactly two (non-degenerate) annuli swept out by closed horizontal trajectories of $\varphi$. Then the Carathéodory and Teichmüller metrics agree on the Teichmüller disc $\tau^{\varphi}$ if and only if the function $\boldsymbol{\Phi}: \mathcal{E}\left(\mathbb{H}^{2}\right) \rightarrow \mathbb{H}$ given by

$$
\boldsymbol{\Phi}(\tau)=\alpha_{1} \lambda_{1}+\alpha_{2} \lambda_{2},
$$

where $\tau=\mathcal{E}\left(\lambda_{1}, \lambda_{2}\right)$, can be extended to a holomorphic function $\boldsymbol{\Phi}: \mathcal{T}_{g, n} \rightarrow \mathbb{H}$.

Remark. In fact, if such a function $\boldsymbol{\Phi}$ exists it has to be unique since $\mathcal{E}\left(\mathbb{H}^{2}\right)$ has non-empty interior. We do not make a use of this fact and we omit its proof.

\section{Translation Equivariant holomorphic functions $\mathbb{H}^{2} \rightarrow \mathbb{H}$}

The main result of this section is the backbone of this paper. However, it is entirely self contained and except the application of its main result it is otherwise not connected to the rest of the paper. The reader may choose to skip it at first reading.

6.1. Rigidity of equivariant holomorphic functions. We are interested in holomorphic functions $f: \mathbb{H}^{2} \rightarrow \mathbb{H}$, satisfying the conditions

$$
\begin{aligned}
& f(\lambda, \lambda)=\lambda \\
& \frac{\partial f}{\partial z}(\lambda, \lambda)=\alpha, \\
& \frac{\partial f}{\partial w}(\lambda, \lambda)=1-\alpha,
\end{aligned}
$$

for every $\lambda \in \mathbb{H}$ and some constant $0<\alpha<1$.

Examples of such maps are

$$
\mathbf{f}_{\alpha}(z, w)=\alpha z+(1-\alpha) w,
$$

where $(z, w)$ denote the coordinates on $\mathbb{H}^{2}$. But there are other such maps. For simplicity we adopt the notation $\mathbf{f}_{\frac{1}{2}}=\mathbf{f}$.

Example. Let

$$
h(z, w)=\frac{1}{2}(z+w)-\frac{1}{2} \frac{(z-w)^{2}}{(z+w)},
$$

The function $h$ maps $\mathbb{H}^{2}$ to $\mathbb{H}$ (this is rather easy to check) and obviously satisfies the three conditions (18), (19),(20), for $\alpha=1 / 2$. Thus, we have found further examples (beside f) satisfying these 3 conditions. But $h$ is interesting for another reason. A map $A: \mathbb{H} \rightarrow \mathbb{H}^{2}$ is called a balanced disc if it is of the form $A=\left(A_{1}, A_{2}\right)$, for some automorphisms $A_{1}, A_{2} \in \operatorname{Aut}(\mathbb{H})$. An extremal disc for a holomorphic map 
$g: \mathbb{H}^{2} \rightarrow \mathbb{H}$ is any balanced disc such that $g \circ A \in \operatorname{Aut}(\mathbb{H})$. The set of points in $\mathbb{H}^{2}$ which are contained in some extremal disc is denoted by $X(g)$. In [14] Knese described all functions $g: \mathbb{H}^{2} \rightarrow \mathbb{H}$, such that $X(g)=\mathbb{H}^{2}$ (our functions $\mathbf{f}_{\alpha}$ are such examples). Furthermore, he constructed examples of functions having two extremal discs but that are not everywhere extremal. One can verify that $X(h)$ contains a two (real) dimensional family of extremal discs (the set $X(h)$ has real dimension three), but $h$ is not everywhere extremal.

However, if we impose an additional translation invariance assumption then functions $\mathbf{f}_{\alpha}$ are the only examples. The following is the main result of this section and it confirms Theorem 5.1 above.

Theorem 6.1. Suppose $f: \mathbb{H}^{2} \rightarrow \mathbb{H}$ satisfies the conditions (18), (19), (20) and

$$
f(z+c, w+c)=f(z, w)+c,
$$

for $(z, w) \in \mathbb{H}^{2}$ and some $c>0$. Then $f \equiv \mathbf{f}_{\alpha}$.

Remark. In fact, a stronger result holds true. Let $f: \mathbb{H}^{2} \rightarrow \mathbb{H}$ be any function that satisfies the conditions (18), (19), (20), but not necessarily the invariance condition (21). Consider the "translation" flow $f_{s}(z, w)=f(z+s, w+s)-s$. It can be proved that for a random choice of the variable $s$ the function $f_{s}$ is very close to being equal to $\mathbf{f}_{\alpha}$. More precisely, if $s \in[-n, n]$ and $\epsilon>0$, then $\left\|f_{s}-\mathbf{f}_{\alpha}\right\| \leq \epsilon$ with the probability $1-O\left(\frac{1}{\epsilon n}\right)$ (where $\left\|f_{s}-\mathbf{f}_{\alpha}\right\|$ is the "weak" distance generated by the topology of convergence on compact sets). This stronger result yields Theorem 6.1.

In the remainder of this section we prove Theorem 6.1.

6.2. Consequences of the Schwarz lemma. The classical Schwarz lemma says that a holomorphic map between complex manifolds does not increase the Carathèodory distance. We are interested in maps $\mathbb{H}^{2} \rightarrow \mathbb{H}$, so first we describe the Carathèodory distances on these two domains.

The Carathèodory distance on $\mathbb{H}$ is equal to the hyperbolic distance $d_{\mathbb{H}}$. We let

$$
d_{\mathbb{H}^{2}}\left(\left(z_{1}, w_{1}\right),\left(z_{2}, w_{2}\right)\right)=\max \left\{d_{\mathbb{H}}\left(z_{1}, z_{2}\right), d_{\mathbb{H}}\left(w_{1}, w_{2}\right)\right\} .
$$

This is the Carathèodory distance on $\mathbb{H}^{2}$.

Let

$$
f_{t}(z, w)=\frac{f(t z, t w)}{t}, \quad t>0 .
$$

Recall $\mathbf{f}(z, w)=\frac{1}{2}(z+w)$. The following lemma plays a key role in the proof of Theorem 6.1.

Lemma 6.1. Suppose $f: \mathbb{H}^{2} \rightarrow \mathbb{H}$, is such that

$$
\lim _{t \rightarrow \infty} f_{t}(z, w)=\mathbf{f}(z, w),
$$

for every $(z, w) \in \mathbb{H}^{2}$. Then

$$
f(\lambda,(2 \mathbf{i}-\lambda))=\mathbf{i},
$$

for every complex number $|\lambda-\mathbf{i}|<1$. 


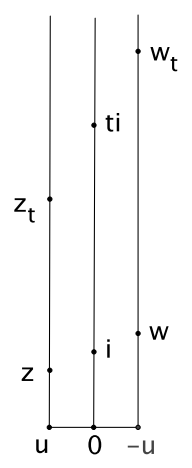

Figure 2. The points $z, w, z_{t}, w_{t}$ for $t=4$

Proof. Suppose $|\lambda-\mathbf{i}|<1$ and $\lambda=u+v \mathbf{i}$. For $t>1$ we let (see Figure 2)

$$
z=u+v \mathbf{i} \quad z_{t}=u+t v \mathbf{i}
$$

and

$$
w=-u+(2-v) \mathbf{i} \quad w_{t}=-u+t(2-v) \mathbf{i} .
$$

One reason behind the definition of $z_{t}$ and $w_{t}$ is the equality

$$
d_{\mathbb{H}^{2}}\left((z, w),\left(z_{t}, w_{t}\right)\right)=d_{\mathbb{H}}(\mathbf{i}, t \mathbf{i}) .
$$

The Schwarz lemma tells us

$$
d_{\mathbb{H}}\left(f(z, w), f\left(z_{t}, w_{t}\right)\right) \leq d_{\mathbb{H}^{2}}\left((z, w),\left(z_{t}, w_{t}\right)\right) .
$$

Together, the previous two equations yield the inequality

$$
d_{\mathbb{H}}\left(f(z, w), f\left(z_{t}, w_{t}\right)\right) \leq d_{\mathbb{H}}(\mathbf{i}, t \mathbf{i}) .
$$

Next, we prove the claim

$$
d_{\mathbb{H}}\left(f\left(z_{t}, w_{t}\right), t \mathbf{i}\right) \rightarrow 0, \quad t \rightarrow \infty .
$$

Indeed, since elements of Aut $(\mathbb{H})$ are isometries of $d_{\mathbb{H}}$, we have

$$
d_{\mathbb{H}}\left(f\left(z_{t}, w_{t}\right), \mathbf{t i}\right)=d_{\mathbb{H}}\left(t^{-1} f\left(z_{t}, w_{t}\right), \mathbf{i}\right) .
$$

Substituting $z_{t}=t\left(t^{-1} z_{t}\right)$ and $w_{t}=t\left(t^{-1} w_{t}\right)$ we get

$$
d_{\mathbb{H}}\left(f\left(z_{t}, w_{t}\right), t \mathbf{i}\right)=d_{\mathbb{H}}\left(f_{t}\left(\left(t^{-1} z_{t}\right),\left(t^{-1} w_{t}\right)\right), \mathbf{i}\right) .
$$

By the assumption of the lemma we have $f_{t} \rightarrow \mathbf{f}$ (uniformly on compact sets). Since

$$
\left(t^{-1} z_{t}, t^{-1} w_{t}\right) \rightarrow(v \mathbf{i},(2-v) \mathbf{i})
$$


we conclude

$$
d_{\mathbb{H}}\left(f\left(z_{t}, w_{t}\right), t \mathbf{i}\right) \rightarrow d_{\mathbb{H}}(\mathbf{f}(v \mathbf{i},(2-v) \mathbf{i}), \mathbf{i}) .
$$

But $\mathbf{f}(v \mathbf{i},(2-v) \mathbf{i})=\mathbf{i}$, so $d_{\mathbb{H}}(\mathbf{f}(v \mathbf{i},(2-v) \mathbf{i}), \mathbf{i})=0$ and $(24)$ is proved.

We apply the triangle inequality and get

$$
d_{\mathbb{H}}(f(z, w), t \mathbf{i}) \leq d_{\mathbb{H}}\left(f(z, w), f\left(z_{t}, w_{t}\right)\right)+d_{\mathbb{H}}\left(f\left(z_{t}, w_{t}\right), t \mathbf{i}\right) .
$$

Combining (23) and (24) we obtain

$$
d_{\mathbb{H}}(f(z, w), t \mathbf{i}) \leq d_{\mathbb{H}}(\mathbf{i}, t \mathbf{i})+o(1),
$$

where $o(1) \rightarrow 0$, when $t \rightarrow \infty$.

When $t \rightarrow \infty$, the family of metric balls of radius $\left(d_{\mathbb{H}}(\mathbf{i}, t \mathbf{i})+o(1)\right)$, and centered at $t \mathbf{i}$, converges to the closed horoball

$$
\mathcal{H}=\{\zeta \in \mathbb{H}: \operatorname{Im}(\zeta) \geq 1\} .
$$

Since the point $f(z, w)$ lives in all these balls, it follows that $f(z, w)$ belongs to $\mathcal{H}$. In terms of the parameter $\lambda$ (recall $z=\lambda$ and $w=2 \mathbf{i}-\lambda$ ) this means that the point $f(\lambda, 2 \mathbf{i}-\lambda) \in \mathcal{H}$, for every $|\lambda-\mathbf{i}|<1$.

Next, let $h$ denote the function $h(\lambda)=(\lambda, 2 \mathbf{i}-\lambda)$, defined on the disc $|\lambda-\mathbf{i}|<1$. Consider the composition $f \circ h$. One one hand, we have $h(\mathbf{i})=(\mathbf{i}, \mathbf{i})$ and thus $(f \circ h)(\mathbf{i})=\mathbf{i}$. On the other hand, we have shown that $f(\lambda, 2 \mathbf{i}-\lambda) \in \mathcal{H}$. But the point $\mathbf{i}$ is on the boundary of $\mathcal{H}$ and from the open mapping theorem we conclude that $h$ is the constant map $(f \circ h) \equiv \mathbf{i}$. We have proved the lemma.

6.3. The proof of Theorem 6.1. First we show that we may assume $\alpha=1 / 2$. Consider the map

$$
\widehat{f}(z, w)=\frac{1}{2}\left(f(z, w)+\mathbf{f}_{1-\alpha}\right) .
$$

Then $\widehat{f}(z, w)$ satisfies (18), (21). It also satisfies the identities (19), (20) for $\alpha=\frac{1}{2}$. If we prove $\widehat{f} \equiv \mathbf{f}$ then (by the linearity of $\mathbf{f}_{\alpha}$ 's in terms of $\alpha$ ) it follows $f \equiv \mathbf{f}_{\alpha}$. Thus in the remainder of the proof we assume $\alpha=1 / 2$ and it remains to prove $f \equiv \mathbf{f}$.

Next, we show that the assumption in Lemma 6.1 is satisfied when $f$ is translation invariant.

Proposition 6.1. Suppose $f: \mathbb{H}^{2} \rightarrow \mathbb{H}$ satisfies the conditions (18), (19), (20) and (21) (assuming that in (19), (20), the parameter $\alpha=\frac{1}{2}$ ). Then

$$
\lim _{t \rightarrow \infty} f_{t}(z, w)=\mathbf{f}(z, w), \quad(z, w) \in \mathbb{H}^{2} .
$$

Proof. Note that $f_{t}$ also satisfies (18), (19) and (20). Moreover

$$
f_{t}\left(z+\frac{c}{t}, w+\frac{c}{t}\right)=f_{t}(z, w)+\frac{c}{t} .
$$

Let $h: \mathbb{H}^{2} \rightarrow \mathbb{H}$ be any accumulation point of the sequence $f_{t}$. Then $h$ satisfies (18), (19) and (20). Moreover, it follows from (26) that

$$
h(z+s, w+s)=h(z, w)+s, \text { for every } s \in \mathbb{R} .
$$

This last translation invariance condition together with the assumption that the range of the map $h$ is the upper half plane $\mathbb{H}$ implies that $h$ is an affine holomorphic 
map of the form $h(z, w)=a z+b w$, for some $a, b \in \mathbb{C}$. We prove this claim in Lemma 11.1 in the second appendix below.

From (19) and (20) we find that $a=b=1 / 2$ and thus $h \equiv \mathbf{f}$. We are done.

We can now complete the proof of Theorem 6.1. Observe that for every $r \in \mathbb{R}$ the map $f(z-r, w-r)+r$ satisfies the conditions (18), (19), (20) and (21). Applying first Proposition 6.1 and then Lemma 6.1 to the map $f(z-r, w-r)+r$, we derive the identity

$$
f(r+\lambda, r+(2 \mathbf{i}-\lambda))=r+\mathbf{i},
$$

for every $r \in \mathbb{R}$ and $|\lambda-\mathbf{i}|<1$. From here we prove Theorem 6.1 , that is we prove $f_{\alpha}=\mathbf{f}$.

The proof of Theorem 6.1 now follows by polarizing the map $f$. More precisely, below we apply the following cornerstone principle from complex analysis (see page 10 in [4] or [14]).

Polarization Principle. Let $\Omega$ be a neighborhood of the origin $(0,0) \in \mathbb{C}^{2}$ and $F: \Omega \rightarrow \mathbb{C}$ a holomorphic map which satisfies the identity $F(z, \bar{z})=0$, for every $(z, \bar{z}) \in \Omega$. Then $F$ is the constant map $F \equiv 0$.

We let $h(z, w)=f(z+\mathbf{i}, w+\mathbf{i})-\mathbf{i}$. The $h$ is defined on the set $\{(z, w):-1<$ $\operatorname{Im}(z), \operatorname{Im}(w)\}$. Choosing $\lambda=(1+s) \mathbf{i}$, for some $s \in(-1,1)$, we deduce from $(27)$ the identity $h(r+\mathbf{i} s, r-\mathbf{i} s)=r$, for every $r \in \mathbb{R}$ and $s \in(-1,1)$. We can write this as $h(\zeta, \bar{\zeta})=r$ for $\zeta \in \Omega=\{(z, w):-1<\operatorname{Im}(z), \operatorname{Im}(w)<-1\}$.

Set $F=h-\mathbf{f}$. We have just shown $h(\zeta, \bar{\zeta})=r$ for $\zeta \in \Omega$. The same holds true for $\mathbf{f}$ and thus the identity $F(\zeta, \bar{\zeta})=0$ holds for every $\zeta \in \Omega$. From the above principle we conclude $F \equiv 0$, that is $h \equiv \mathbf{f}$. This implies $f \equiv \mathbf{f}$ and the proof is complete.

\section{L-SHAPED PILLOWCASES}

7.1. Definitions. We make the standing assumption that $a>0$ and $0<q<1$. Unless otherwise stated we assume $b>0$. Let $L=L(a, b, q)$ denote the $L$-shaped polygon as in Figure 3. By $P_{k}, k=1, \ldots, 5$, we denote the vertices at which the interior angle is $\pi / 2$ and by $Q$ the remaining vertex at which the interior angle is $3 \pi / 2$.

Let $S=S(a, b, q)$ denote the double of the polygon $L$. Formally $S$ is defined as a half-translation surface. As a Riemann surface $S$ is a five times punctured Riemann sphere ( $P_{k}$ 's are the marked points). We call $S$ an L-shaped pillowcase. (If we allow $b=0$ then $S$ is still a well defined Riemann surface. In this case $S$ is a pillowcase surface with the additional marked point $P_{3}$ that lives on the edge $\left.P_{2} P_{4}\right)$.

The $(2,0)$ form $d z^{2}$ lives on the polygon $L$. After doubling, the two copies of the form $d z^{2}$ (one on each copy of $L$ ) glue together along the edges of the L-shaped pillowcase $S$ to form the holomorphic quadratic differential $\psi=\psi(a, b, q)$ on $S$. The differential $\psi$ has the first order poles at the points $P_{k}$ and the first order zero at $Q \in S$. Moreover, $\psi$ is a J-S differential and $S$ decomposes into two non-degenerate annuli $\Pi_{1}$ and $\Pi_{2}$ swept out by closed horizontal trajectories of $\psi$. (If $b=0$ then $\psi$ has first order poles at the points $P_{1}, P_{2}, P_{4}, P_{5}$ and no zeroes). 


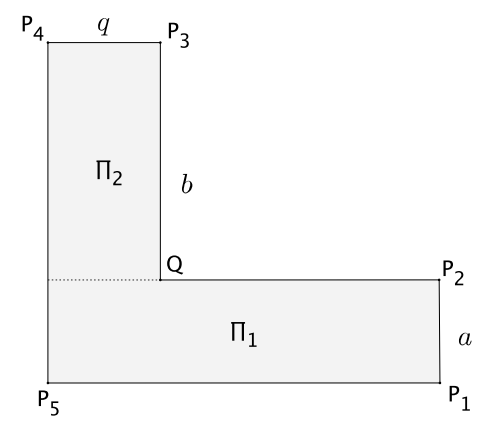

FigURE 3. $L$-shaped polygon $L(a, b, q)$

Remark. The differential $\psi$ is not a square of an Abelian differential. The corresponding degree two branched cover, where the lift of $\psi$ (which we denote by $\widehat{\psi}$ ) is a square of an Abelian differential, is a genus two surface and the Abelian differential $\sqrt{\widehat{\psi}}$ is a Weierstrass form (see [18]).

We consider surfaces $S(a, b, q)$ as marked surfaces, that is as elements of $\mathcal{T}_{0,5}$. Once and for all, we fix a marking such that the surfaces $S(a, b, q)$ are simultaneously marked, that is the corresponding family of marked surfaces $S(a, b, q)$ varies continuously in $\mathcal{T}_{0,5}$ when $a, b, q$ vary continuously.

The family of pairs $(S(a, b, q), \psi(a, b, q))$ is a real 3 -dimensional locus of the cotangent bundle over $\mathcal{T}_{0,5}$ while the family $S(a, b, q)$ is a real 2 -dimensional locus in $\mathcal{T}_{0,5}$. But, deciding when two $L$-shaped pillowcases are the same as marked surfaces is not so easy. If the marked surfaces $S\left(a_{1}, b_{1}, q_{1}\right)$ and $S\left(a_{2}, b_{2}, q_{2}\right)$ represent the same point in $\mathcal{T}_{0,5}$ then the corresponding triples are related by certain nonelementary functions arising from the Schwarz-Christoffel formula for conformal maps from the unit disc onto $L$-shaped polygons.

7.2. The stabilizer of the Teichmüller disc $\tau^{\psi(a, b, q)}$. By $\gamma_{1}$ and $\gamma_{2}$ we denote simple closed curves homotopic to $\Pi_{1}$ and $\Pi_{2}$ respectively. . The modulus of the annulus $\Pi_{1}$ is $m_{1}=b / 2 q$ and of $\Pi_{2}$ is $m_{2}=a / 2$ (the moduli of the corresponding rectangle $\Pi_{j}$ is $2 m_{j}$ ).

Assuming $m_{1} / m_{2}$ is rational it follows that $\psi(a, b, q)$ is a rational J-S differential. We let

$$
t=\operatorname{lcm}\left\{\frac{1}{m_{1}}, \frac{1}{m_{2}}\right\}=\operatorname{lcm}\left\{\frac{2 q}{b}, \frac{2}{a}\right\} .
$$

By Proposition 2.1 we know that $A_{t} \in \operatorname{Stab}\left(\tau^{\psi}\right)$ where $A_{t}(\lambda)=\lambda+t, \lambda \in \mathbb{H}$. Moreover, $\tau_{*}^{\psi}(A)=T \in \operatorname{Mod}_{0,5}$ is the product of the (commuting) Dehn twists $T=T_{\gamma_{1}}^{n_{1}} T_{\gamma_{2}}^{n_{2}}$ where $n_{j}=m_{j} t$.

7.3. The Carathéodory metric on $\tau^{\psi(a, b, q)}$. Fix a triple $\left(a_{0}, b_{0}, q_{0}\right)$ and let $\psi_{0}=$ $\psi\left(a_{0}, b_{0}, q_{0}\right)$. We let $\mathcal{E}_{0}: \mathbb{H}^{2} \rightarrow \mathcal{T}_{0,5}$ be the poly-plane mapping corresponding to 
$\psi_{0}$. Also, set

$$
\alpha_{1}=\frac{1}{\left\|\psi_{0}\right\|_{1}} \int_{\Pi_{1}}\left|\psi_{0}\right|=\frac{b_{0} q_{0}}{a_{0}+b_{0} q_{0}}
$$

and

$$
\alpha_{2}=\frac{1}{\left\|\psi_{0}\right\|_{1}} \int_{\Pi_{2}}\left|\psi_{0}\right|=\frac{a_{0}}{a_{0}+b_{0} q_{0}} .
$$

If $a_{0}, b_{0}, q_{0} \in \mathbb{Q}$ and $\tau^{\psi_{0}}$ is an extremal disc for some holomorphic function $\mathcal{T}_{0,5} \rightarrow \mathbb{H}$ then by Theorem 5.1 there exists a holomorphic function $\boldsymbol{\Phi}: \mathcal{T}_{0,5} \rightarrow \mathbb{H}$ such that

$$
\boldsymbol{\Phi}\left(\mathcal{E}_{0}\left(\lambda_{1}, \lambda_{2}\right)\right)=\alpha_{1} \lambda_{1}+\alpha_{2} \lambda_{2},
$$

where $\left(\lambda_{1}, \lambda_{2}\right) \in \mathbb{H}^{2}$. We find that for any $a$ and $b$ the equalty

$$
\mathcal{E}_{0}\left(\frac{b}{b_{0}} \mathbf{i}, \frac{a}{a_{0}} \mathbf{i}\right)=S\left(a, b, q_{0}\right)
$$

holds. The last two formulas yield the following equality

$$
\boldsymbol{\Phi}\left(S\left(a, b, q_{0}\right)\right)=\left(\frac{a+b q_{0}}{a_{0}+b_{0} q_{0}}\right) \mathbf{i} .
$$

Thus, we have found a holomorphic function $\mathbf{\Phi}$ whose restriction to the locus of surfaces $S\left(a, b, q_{0}\right)$ is given by $(28)$. As it turns out, up to multiplication by a constant the restriction of $\boldsymbol{\Phi}$ to the locus $S\left(a, b, q_{0}\right)$ depends only on $q_{0}$.

Lemma 7.1. Fix $q_{0} \in(0,1)$ and suppose that the Carathéodory and Teichmüller metrics agree on $\tau^{\psi\left(a_{0}, b_{0}, q_{0}\right)}$ for some choice of $a_{0}, b_{0}>0$. Then there exists a holomorphic function $\boldsymbol{\Psi}: \mathcal{T}_{0,5} \rightarrow \mathbb{H}$ such that

$$
\boldsymbol{\Psi}\left(S\left(a, b, q_{0}\right)\right)=\left(a+b q_{0}\right) \mathbf{i}
$$

for every $a>0$ and $b \geq 0$.

Proof. We let

$$
\boldsymbol{\Psi}=\frac{\boldsymbol{\Phi}}{a_{0}+q_{0} b_{0}} .
$$

Then $\boldsymbol{\Psi}: \mathcal{T}_{0,5}$ is holomorphic and from (28) we see that $\boldsymbol{\Psi}$ satisfies the stated equality. To derive this formula we assumed $b>0$. But since $S(a, b, q) \rightarrow S(a, 0, q)$ in $\mathcal{T}_{0,5}$ when $b \rightarrow 0$, the formula also holds for $b=0$. (The formula does not hold for $a=0$ since the surface $S(0, b, q)$ is not well defined, that is $S(a, b, q)$ tends to $\infty$ in $\mathcal{T}_{0,5}$ when $\left.a \rightarrow \infty\right)$.

Remark. It is well known that the Carathéodory and Teichmüller metrics agree on the disc $\tau^{\psi\left(a, 0, q_{0}\right)}$. In fact $\tau^{\psi\left(a, 0, q_{0}\right)}$ is an extremal disc for the forgetful map $\pi: \mathcal{T}_{0,5} \rightarrow \mathcal{T}_{0,4} \cong \mathbb{H}$ (see [2] and [6]). But each disc $\tau^{\psi\left(a, b, q_{0}\right)}$, when $b>0$, is extremal for $\boldsymbol{\Psi}$ and by continuity so is the $\operatorname{disc} \tau^{\psi(a, 0, q)}$. It is easy to see that $\pi$ and $\boldsymbol{\Psi}$ (if it exists) are different holomorphic functions (for example, none of the $\operatorname{discs} \psi\left(a, b, q_{0}\right)$, when $b>0$, is extremal for $\left.\pi\right)$. 


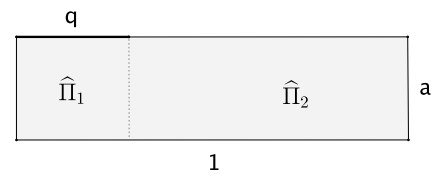

Figure 4. The annuli $\widehat{\Pi}_{j}$

\section{The Proof of Theorem 1.1}

Fix a pillowcase $S=S(a, 0, q)$ for some $a, q$. For small $t>0$ we let $S(t)=$ $S(a, 0, q-t)$. Next we show that $S(t)$ is a $C^{\infty}$ path in $\mathcal{T}_{0,5}$. Our aim is to prove that $\boldsymbol{\Psi}$ (corresponding to $q$ ) from Lemma 7.1 is not smooth along the path $S(t)$ at $t=0$. This implies that the Carathéodory and Teichmüller metrics do not agree.

Lemma 8.1. The path $S(t)$ is infinitely differentiable.

Proof. The path $S(t)$ can be defined as $S(t)=[\mu(t)]$ where $\mu(t) \in \mathcal{B D}_{1}(S)$ is equal to $\mu_{j}(t)$ on the annulus $\widehat{\Pi}_{j}$ (see Figure 4 )

$$
\mu_{1}(t)=\frac{-t}{2 q-t}, \quad \mu_{2}(t)=\frac{t}{2(1-q)+t} .
$$

The Beltrami dilatation $\mu(t)$ is pointwise smooth function of $t$ and thus $S(t)$ is a smooth path in $\mathcal{T}_{0,5}$.

Assume now that the Carathéodory and Teichmüller metrics agree on the disc $\tau^{\psi(a, b, q)}$, for some choice of $b>0$. Let $\boldsymbol{\Psi}: \mathcal{T}_{0,5} \rightarrow \mathbb{H}$ be the function from Lemma 7.1 corresponding to $q$.

The function $\boldsymbol{\Psi}$ is holomorphic and the path $S(t)$ smooth, so the function $\Psi(S(t))$ is a complex valued smooth function of $t$. Thus

$$
\boldsymbol{\Psi}(S(t))=\boldsymbol{\Psi}(S(0))+\delta_{1} t+\delta_{2} t^{2}+O\left(t^{3}\right),
$$

for some constants $\delta_{1}, \delta_{2} \in \mathbb{C}$.

Using the explicit formula for $\boldsymbol{\Psi}$ from Lemma 7.1, in the next section we prove the following lemma.

Lemma 8.2. Let $a, q, q_{1}$ such that $q>q_{1}$ and denote by $\boldsymbol{\Psi}$ the holomorphic function from Lemma 7.1 corresponding to $q$. Then there are constants $\beta_{1}$ and $\beta_{2} \neq 0$ such that

$\boldsymbol{\Psi}\left(S\left(a, 0, q_{1}\right)\right)=\boldsymbol{\Psi}(S(a, 0, q))+\beta_{1}(1+o(1)) \frac{t}{\log t^{-1}}+\beta_{2}(1+o(1)) \frac{t^{2}}{\log t^{-1}}+o\left(\frac{t^{2}}{\log t^{-1}}\right)$, where $t=q-q_{1}$ and $o(1) \rightarrow 0$ when $t \rightarrow 0$. 
Applying this lemma to our situation (and letting $q_{1}=q-t$ ) gives the equality

$$
\boldsymbol{\Psi}(S(t))=\boldsymbol{\Psi}(S(0))+\beta_{1}(1+o(1)) \frac{t}{\log t^{-1}}+\beta_{2}(1+o(1)) \frac{t^{2}}{\log t^{-1}}+o\left(\frac{t^{2}}{\log t^{-1}}\right) .
$$

This equality and the equality (29) offer two competing expressions for $\boldsymbol{\Psi}(S(t))$ $\boldsymbol{\Psi}(S(0))$. Equating them gives

$$
\delta_{1} t+\delta_{2} t^{2}+O\left(t^{3}\right)=\beta_{1}(1+o(1)) \frac{t}{\log t^{-1}}+\beta_{2}(1+o(1)) \frac{t^{2}}{\log t^{-1}}+o\left(\frac{t^{2}}{\log t^{-1}}\right) .
$$

Using (30), we first check that $\delta_{1}=0$ since otherwise the left hand side would be larger than the right hand side for $t$ small enough. Second we see that $\beta_{1}=0$ since otherwise the right hand side would be larger than the left hand side for $t$ small enough. The equality (30) becomes

$$
\delta_{2} t^{2}+O\left(t^{3}\right)=\beta_{2}(1+o(1)) \frac{t^{2}}{\log t^{-1}}+o\left(\frac{t^{2}}{\log t^{-1}}\right) .
$$

Similarly we see that $\delta_{2}=0$ since otherwise the left hand side would be larger than the right hand side for $t$ small enough. We are down to

$$
O\left(t^{3}\right)=\beta_{2}(1+o(1)) \frac{t^{2}}{\log t^{-1}}+o\left(\frac{t^{2}}{\log t^{-1}}\right) .
$$

Divide the both sides of the equality by $\frac{t^{2}}{\log t^{-1}}$ and get

$$
O\left(t \log t^{-1}\right)=\beta_{2}(1+o(1))+o(1) .
$$

Since $\beta_{2} \neq 0$ we derive a contradiction and Theorem 1.1 is proved.

\section{Endgame: The Proof of Lemma 8.2}

9.1. The Schwarz-Christoffel maps. Let $w_{1}, w_{2}, w_{3}, w_{4}, w_{5}, \eta$ denote points on the extended real line such that $w_{1}=\infty, w_{2}=-1, w_{3}=\zeta, w_{4}=\lambda, w_{5}=1$, where $-1<\eta \leq \zeta<\lambda<1$. Consider the map $F(\lambda, \zeta, \eta)=F: \mathbb{H} \rightarrow \mathbb{C}$ given by

$$
F(z)=J^{-1} \int_{0}^{z} \frac{\sqrt{w-\eta}}{\sqrt{w+1} \sqrt{w-\zeta} \sqrt{w-\lambda} \sqrt{w-1}} d w
$$

for $z \in \mathbb{H}$ and some constant $J>0$.

In general $F$ does not have to be injective but in this case it always is. Observe that $F$ maps $\overline{\mathbb{R}}$ onto a closed hexagonal chain of lines that has 5 interior angles equal to $\frac{\pi}{2}$ (at the points $F\left(w_{k}\right)$ ) and one interior angle equal $\frac{3 \pi}{2}$ (at $F(\eta)$ ). Every such hexagonal chain (with this choice of angles) must be embedded in the plane and we conclude that $F$ is injective. The image $F(\mathbb{H})$ is an $L$-shaped polygon. As usual, we refer to $F$ as the Schwarz-Christoffel map.

From the standard theorem in complex analysis we know that for any triple $(a, b, q)$ we can find the unique $-1<\eta \leq \zeta<\lambda<1$ and $J>0$ such that the corresponding Schwarz-Christoffel map $F$ maps $\mathbb{H}$ onto $L(a, b, q)$, that is $F: \mathbb{H} \rightarrow$ $L(a, b, q)$ and $F\left(w_{j}\right)=P_{j}$ and $F(\eta)=Q$. We note that $b=0$ if and only if $\eta=\zeta$. 
By definition, the bottom horizontal side $\overline{P_{5} P_{1}}$ of the polygon $L(a, b, q)$ is of length one. This enables us to express the constant $J$ as

$$
J=\int_{1}^{\infty} \frac{\sqrt{x-\eta}}{\sqrt{x+1} \sqrt{x-\zeta} \sqrt{x-\lambda} \sqrt{x-1}} d x .
$$

The pillowcase $S=S(a, b, q)$ is the double of $L=L(a, b, q)$. Note the anticonformal involution of $S$ that exchanges the two copies of $L$ and point-wise fixes the (common) boundary of the two copies of $L$. Now we extend the map $F$ to the lower half plane so that it maps the lower half plane onto the second copy of $L$. This extended map (we use $F$ to denoted the extension) $F: \mathbb{C} \backslash\{-1, \zeta, \lambda, 1\} \rightarrow S(a, b, q)$ is conformal and $F(\eta)=Q$. Moreover, $F$ conjugates the anti-conformal involution $z \rightarrow \bar{z}$ of $\mathbb{C}$ to the anti-conformal involution on $S$.

9.2. Computing the Schwarz-Christoffel maps. Fix $-1<\eta=\zeta<\lambda<1$. For small $r>0$ we let $F(\lambda, \zeta, \zeta-r)=F$ denote the corresponding SchwarzChristoffel map. Varying $r$ yields the family of conformal mappings $F: X(\lambda, \zeta) \rightarrow$ $S(a(r), b(r), q(r))$ for suitable $a(r), b(r), q(r)$. Thus, we get an 1-dimensional family of $L$-pillowcases $S(r)=S(a(r), b(r), q(r))$ that are equal to each other in $\mathcal{T}_{0,5}$. Note that $b(0)=0$ and that $q(r)$ is an increasing function.

Our goal is to compute $a(r), b(r)$ and $q(r)$. We want to establish the following equality

$$
\left.a(r)+b(r) q(r)=1+D r+C r^{2} \log r^{-1}+o\left(r^{2} \log r^{-1}\right)\right)
$$

where $D$ and $C$ are constants such that $C \neq 0$. This is the content of Proposition 9.4 below. From there we easily derive Lemma 8.2 which is the goal of this section.

We compute $a(r), b(r)$ and $q(r)$ from the formula for the Schwarz-Christoffel map. We find

where

$$
a(r)=\frac{A(r)}{J(r)}, \quad b(r)=\frac{B(r)}{J(r)}, \quad q(r)=\frac{Q(r)}{J(r)},
$$

$$
\begin{aligned}
& A(r)=-\mathbf{i} \int_{-\infty}^{-1} \frac{\sqrt{x-\zeta+r}}{\sqrt{x+1} \sqrt{x-\zeta} \sqrt{x-\lambda} \sqrt{x-1}} d x \\
& B(r)=-\mathbf{i} \int_{\zeta-r}^{\zeta} \frac{\sqrt{x-\zeta+r}}{\sqrt{x+1} \sqrt{x-\zeta} \sqrt{x-\lambda} \sqrt{x-1}} d x \\
& J(r)=\int_{1}^{\infty} \frac{\sqrt{x-\zeta+r}}{\sqrt{x+1} \sqrt{x-\zeta} \sqrt{x-\lambda} \sqrt{x-1}} d x, \\
& Q(r)=-\int_{\zeta}^{\lambda} \frac{\sqrt{x-\zeta+r}}{\sqrt{x+1} \sqrt{x-\zeta} \sqrt{x-\lambda} \sqrt{x-1}} d x .
\end{aligned}
$$

Observe $A(r), J(r), Q(r)>0$ for every $r$ and $B(r)>0$ for $r>0$. We first analyze these functions. Then in the next subsection we draw the conclusions about $a(r), b(r)$ and $q(r)$. 
Functions $A(r)$ and $J(r)$ are in fact real analytic for $r$ sufficiently small since the corresponding integrand functions depend analytically on $r$ and are uniformly integrable. As for the function $B(r)$ we have the following proposition.

Proposition 9.1. We have

$$
B(r)=\frac{\pi r}{2 \sqrt{\zeta+1} \sqrt{\lambda-\zeta} \sqrt{1-\zeta}}+O\left(r^{2}\right) .
$$

Proof. The equality

$$
\frac{-\mathbf{i}}{\sqrt{x+1} \sqrt{x-\lambda} \sqrt{x-1}}=\frac{\mathbf{i}+O(r)}{\sqrt{\zeta+1} \sqrt{\lambda-\zeta} \sqrt{1-\zeta}},
$$

holds for every $x \in[\zeta-r, \zeta]$, so we conclude

$$
B(r)=\frac{\mathbf{i}+O(r)}{\sqrt{\zeta+1} \sqrt{\lambda-\zeta} \sqrt{1-\zeta}} \int_{\zeta-r}^{\zeta} \frac{\sqrt{x-\zeta+r}}{\sqrt{x-\zeta}} d x
$$

On the other hand, one computes (recall that $r$ is non-negative)

$$
\text { i } \int_{\zeta-r}^{\zeta} \frac{\sqrt{x-\zeta+r}}{\sqrt{x-\zeta}} d x=\int_{\zeta-r}^{\zeta} \frac{\sqrt{x-\zeta+r}}{\sqrt{\zeta-x}} d x
$$

Substituting $\zeta-x=y$ yields

$$
\begin{aligned}
\int_{\zeta-r}^{\zeta} \frac{\sqrt{x-\zeta+r}}{\sqrt{\zeta-x}} d x & =\int_{0}^{r} \frac{\sqrt{r-y}}{\sqrt{y}} d y \\
& =\left.\left[\sqrt{r-y} \sqrt{y}+r \arctan \left(\frac{\sqrt{y}}{\sqrt{r-y}}\right)\right]\right|_{0} ^{r}=\frac{r \pi}{2},
\end{aligned}
$$

and we obtain the desired equality.

It remains to analyze $Q(t)$.

Proposition 9.2. The following equality holds

$$
Q(r)-Q(0)=\frac{r \log r^{-1}}{2 \sqrt{\zeta+1} \sqrt{\lambda-\zeta} \sqrt{1-\zeta}}+o\left(r \log r^{-1}\right) .
$$

Proof. Let $\epsilon=\epsilon(r)>0$ be any function that tends to 0 when $r \rightarrow 0$. We write

$$
Q(r)-Q(0)=\frac{(1+o(1))}{\sqrt{\zeta+1} \sqrt{\lambda-\zeta} \sqrt{1-\zeta}} \int_{\zeta}^{\zeta+\epsilon}\left(\frac{\sqrt{x-\zeta+r}}{\sqrt{x-\zeta}}-1\right) d x+O\left(\frac{r}{\epsilon}\right),
$$

where $o(1) \rightarrow 0$ when $r \rightarrow 0$. We used two things here. First

$$
-\frac{1}{\sqrt{x+1} \sqrt{x-\lambda} \sqrt{x-1}}=\frac{(1+O(\epsilon))}{\sqrt{\zeta+1} \sqrt{\lambda-\zeta} \sqrt{1-\zeta}},
$$

when $\zeta \leq x \leq \zeta+\epsilon$. We then set $o(1)=O(\epsilon)$. Second

$$
\int_{\zeta+\epsilon}^{\lambda}\left(\frac{\sqrt{x-\zeta+r}}{\sqrt{x-\zeta}}-1\right) \frac{1}{\sqrt{x+1} \sqrt{x-\lambda} \sqrt{x-1}} d x=O\left(\frac{r}{\epsilon}\right)
$$


since

$$
\left(\frac{\sqrt{x-\zeta+r}}{\sqrt{x-\zeta}}-1\right)=O\left(\frac{r}{\epsilon}\right)
$$

when $x \geq \zeta+\epsilon$.

Substituting $x-\zeta=y$ yields

$$
\begin{aligned}
\int_{\zeta}^{\zeta+\epsilon} \frac{\sqrt{x-\zeta+r}}{\sqrt{x-\zeta}} d x=\int_{0}^{\epsilon} \frac{\sqrt{y+r}}{\sqrt{y}} d y & =\left.[\sqrt{y} \sqrt{r+y}+r \log (\sqrt{y}+\sqrt{r+y})]\right|_{0} ^{\epsilon} \\
& =\sqrt{\epsilon} \sqrt{r+\epsilon}+r \log \frac{\sqrt{\epsilon}+\sqrt{r+\epsilon}}{\sqrt{r}}
\end{aligned}
$$

We get

$$
\int_{\zeta}^{\zeta+\epsilon}\left(\frac{\sqrt{x-\zeta+r}}{\sqrt{x-\zeta}}-1\right) d x=O\left(\frac{r}{\epsilon}\right)+r \log \frac{\sqrt{\epsilon}+\sqrt{r+\epsilon}}{\sqrt{r}} .
$$

Let

$$
\epsilon(r)=\frac{1}{\sqrt{\log r^{-1}}}
$$

Then

$$
\begin{aligned}
\int_{\zeta}^{\zeta+\epsilon}\left(\frac{\sqrt{x-\zeta+r}}{\sqrt{x-\zeta}}-1\right) d x & =O\left(r \sqrt{\log r^{-1}}\right)+\left(\frac{1}{2}+o(1)\right) r \log r^{-1} \\
& =\frac{1}{2} r \log r^{-1}+o\left(r \log r^{-1}\right)
\end{aligned}
$$

where $o(1) \rightarrow 0$ when $r \rightarrow 0$. Here we used the equality $O\left(r \sqrt{\log r^{-1}}\right)=$ $o\left(r \log r^{-1}\right)$. We replace this in the formula above and obtain

$$
Q(r)-Q(0)=\frac{(1+o(1))\left(\frac{1}{2} r \log r^{-1}+o\left(r \log r^{-1}\right)\right)}{\sqrt{\zeta+1} \sqrt{\lambda-\zeta} \sqrt{1-\zeta}}+O\left(r \sqrt{\log r^{-1}}\right)
$$

and the desired equality follows.

9.3. Differentiating $a(r), b(r)$ and $q(r)$. We derive corollaries (concerning $a(r), b(r)$ and $q(r)$ ) of the formulae we computed in the previous subsection). Since $A(r)$ and $J(r)$ are smooth and $J(0)>0$ it follows that $a(r)$ is smooth near 0 and thus

$$
a(r)=a(0)+D_{1} r+O\left(r^{2}\right)
$$

for some constant $D_{1}$.

Proposition 9.3. There exists $C_{1}>0$ such that

$$
q(r)-q(0)=C_{1} r \log r^{-1}+o\left(r \log r^{-1}\right) .
$$


Proof. Since $J(r)$ is smooth near 0 and $J(0)>0$ from Proposition 9.2 we find

$$
q(r)-q(0)=\frac{Q(r)}{J(r)}-\frac{Q(0)}{J(0)}=\frac{r \log r^{-1}}{2 J(0) \sqrt{\zeta+1} \sqrt{\lambda-\zeta} \sqrt{1-\zeta}}+o\left(r \log r^{-1}\right) .
$$

Letting

$$
C_{1}=\frac{1}{2 J(0) \sqrt{\zeta+1} \sqrt{\lambda-\zeta} \sqrt{1-\zeta}},
$$

yields the stated formula.

Proposition 9.4. There are constants $D$ and $C>0$ such that

$$
\left.a(r)+b(r) q(r)=a(0)+D r+C r^{2} \log r^{-1}+o\left(r^{2} \log r^{-1}\right)\right) .
$$

Proof. Again, since $J(r)$ is smooth and $J(0)>0$ from Proposition 9.1 we find

$$
b(r)=\frac{B(r)}{J(r)}=D_{2} r+O\left(r^{2}\right)
$$

for

$$
D_{2}=\frac{\pi}{2 J(0) \sqrt{\zeta+1} \sqrt{\lambda-\zeta} \sqrt{1-\zeta}} .
$$

Together with the previous Proposition 9.3 this gives

$$
\begin{aligned}
b(r) q(r) & =\left(D_{2} r+O\left(r^{2}\right)\right)\left(q(0)+C_{1} r \log r^{-1}+o\left(r \log r^{-1}\right)\right) \\
& \left.=q(0) D_{2} r+D_{2} C_{1} r^{2} \log r^{-1}+o\left(r^{2} \log r^{-1}\right)\right) .
\end{aligned}
$$

Combining this with (31) and letting

$$
D=D_{1}+q(0) D_{2}, \quad C=C_{1} D_{2},
$$

proves the proposition (note that both $C_{1}>0$ and $D_{2}>0$ were computed explicitly).

9.4. The proof of Lemma 8.2. We are ready to prove Lemma 8.2. Recall that we need to prove that for some $\beta_{2} \neq 0$ the equality

$$
\boldsymbol{\Psi}\left(S\left(a, 0, q_{1}\right)\right)=\boldsymbol{\Psi}(S(a, 0, q))+\beta_{1}(1+o(1)) \frac{t}{\log t^{-1}}+\beta_{2}(1+o(1)) \frac{t^{2}}{\log t^{-1}}+o\left(\frac{t^{2}}{\log t^{-1}}\right),
$$

holds where $t=q-q_{1}$ and $\boldsymbol{\Psi}=\mathbf{\Psi}(q)$ is the function from Lemma 7.1.

Given $a$ and $q_{1}$ we first find $-1<\zeta<\lambda<1$ such that the Schwarz-Christoffel map $F=F(\lambda, \zeta, \zeta)$ maps $\mathbb{H}$ onto $S\left(a, 0, q_{1}\right)$. We start the $r$ flow $S(a(r), b(r), q(r))$, that is $S(a(r), b(r), q(r))$ is the image of $\mathbb{H}$ under the Schwarz-Christoffel map $F=$ $F(\lambda, \zeta, \zeta-r)$. Observe that $q(r)$ is increasing with $r$.

We locate the time $r>0$ such that $q=q(r)$. From Proposition 9.3 it follows that $q=q(r)$ for some $r$ which satisfies the equality

$$
q-q_{1}=t=C_{1} r \log r^{-1}+o\left(r \log r^{-1}\right),
$$

for $C_{1}>0$. Thus

$$
r=\frac{(1+o(1)) t}{C_{1} \log t^{-1}} \quad \text { and } \quad \log r^{-1}=(1+o(1)) \log t^{-1},
$$

where $o(1) \rightarrow 0$ when $t \rightarrow 0$. 
Since by construction $S\left(a, 0, q_{1}\right)=S(a(r), b(r), q(r))$ in $\mathcal{T}_{0,5}$, and $q=q(r)$, from Lemma 7.1 we find

$$
\boldsymbol{\Psi}\left(S\left(a, 0, q_{1}\right)\right)=\boldsymbol{\Psi}(S(a(r), b(r), q(r)))=\mathbf{i}(a(r)+b(r) q(r)) .
$$

On the other hand, we have $a=a(0)$ and so $\boldsymbol{\Psi}(S(a, 0, q))=\boldsymbol{\Psi}(S(a(0), 0, q))=a(0)$ (again using Lemma 7.1). Together the last two identities imply

$$
\boldsymbol{\Psi}\left(S\left(a, 0, q_{1}\right)\right)-\boldsymbol{\Psi}(S(a, 0, q))=\mathbf{i}(a(r)+b(r) q(r)-a(0)) .
$$

From Proposition 9.4 we get

$$
\left.\boldsymbol{\Psi}\left(S\left(a, 0, q_{1}\right)\right)-\mathbf{\Psi}(S(a, 0, q))=(D \mathbf{i}) r+(C \mathbf{i}) r^{2} \log r^{-1}+o\left(r^{2} \log r^{-1}\right)\right) .
$$

Replacing $r$ with $t$ as in (32) shows that the right hand side in the previous equality is equal to

$$
(D \mathbf{i}) \frac{(1+o(1)) t}{C_{1} \log t^{-1}}+(C \mathbf{i})\left(\frac{(1+o(1)) t}{C_{1} \log t^{-1}}\right)^{2}(1+o(1)) \log t^{-1}+o\left(\frac{t^{2}}{\log t^{-1}}\right) .
$$

Letting

$$
\beta_{1}=\frac{D \mathbf{i}}{C_{1}} \quad \beta_{2}=\frac{C \mathbf{i}}{C_{1}^{2}},
$$

and observing $\beta_{2} \neq 0$ yields Lemma 8.2.

\section{Appendix 1: The proof of Proposition 1.1}

We need to show that $\mathcal{T}_{0,5}$ isometrically and holomorphically embeds into each $\mathcal{T}_{g}, g \geq 2$.

10.1. Signature of a Fuchsian group. Recall that the Euler characteristic of a Fuchsian group $\Gamma$ of signature $\left(g ; k_{1}, \ldots, k_{n}\right)$ is given by

$$
\chi(\Gamma)=2-2 g-\sum_{t=1}^{n}\left(1-\frac{1}{k_{t}}\right) .
$$

Here $k_{t}$ is the order of the corresponding generator of $\Gamma$. If $k_{t}<\infty$ then this generator is elliptic and if $k_{t}=\infty$ then it is parabolic. By $\mathcal{T}\left(g ; k_{1}, \ldots, k_{n}\right)$ we denote the Teichmüller space of $\Gamma$.

We recall the following well known fact: For any choice of $k_{t}$ 's we have that $\mathcal{T}(g ; \infty, \ldots, \infty)$ is biholomorphic to $\mathcal{T}\left(g ; k_{1}, \ldots, k_{n}\right)$, where $\infty$ is repeated $n$ times (see [15]). Since $\mathcal{T}(g ; \infty, \ldots, \infty)$ is biholomorphic to $\mathcal{T}_{g, n}$, we find that

$$
\mathcal{T}_{(g, n)} \simeq \mathcal{T}\left(g ; k_{1}, \ldots, k_{n}\right)
$$

holds for any choice if $k_{i}$ 's, where $\simeq$ denotes the relation of being biholomorphic.

Let $\Gamma_{1}$ be a finite index torsion free subgroup of a Fuchsian group $\Gamma$ of signature $\left(g ; k_{1}, \ldots, k_{n}\right)$. Suppose that each $k_{t}<\infty$. Then $\Gamma$ (and thus $\left.\Gamma_{1}\right)$ has no parabolic elements. This yields that $\Gamma_{1}$ is the fundamental group of a genus $g$ surface for some $g \geq 2$. The Teichmüller space $\mathcal{T}\left(g ; k_{1}, \ldots, k_{n}\right)=\mathcal{T}(\Gamma)$ isometrically and holomorphically embeds into $\mathcal{T}\left(\Gamma_{1}\right) \simeq \mathcal{T}_{g}$. By the Riemann-Hurwitz formula we have

$$
\chi\left(\Gamma_{1}\right)=\chi(\Gamma)\left[\Gamma: \Gamma_{1}\right]
$$

which implies

$$
2 g-2=\chi(\Gamma)\left[\Gamma: \Gamma_{1}\right]
$$


This formula computes the genus of $\Gamma_{1}$ in terms of the Euler characteristic of $\Gamma$ and the index of $\Gamma_{1}$ in $\Gamma$.

10.2. The proof of Proposition 1.1. It was proved in Theorem 1.2 in [8] that a Fuchsian group $\Gamma$ of signature $\left(g ; k_{1}, \ldots, k_{n}\right)$ contains a torsion free subgroup $\Gamma_{1}$ of index $D$ if and only if $D$ is divisible by $2^{\epsilon} \lambda$, where $\lambda$ is the lowest common multiple of $k_{i}$ 's and $\epsilon$ is equal to zero or 1 depending on whether the group $\Gamma$ is of even or odd type (group $\Gamma$ of odd type if $\lambda$ is even, but $\frac{\lambda}{k_{t}}$ is odd for exactly odd number of $i$ 's; otherwise $\Gamma$ is of even type).

Let $\Gamma$ be a Fuchsian group of signature $(0 ; 2,2,2,2,2)$. Then $\Gamma$ is of odd type and by the previous result we see that for every integer $m \geq 1$, the group $\Gamma$ contains

a torsion free subgroup $\Gamma_{m}$ of index $4 m$. Since $\chi(\Gamma)=-\frac{1}{2}$ by (35) it follows that $\chi\left(\Gamma_{m}\right)=-2 m$, and thus $\Gamma_{m}$ is the fundamental group of the closed surface of genus $m+1$. Therefore $\mathcal{T}(\Gamma)$ naturally (and in particular holomorphically and isometrically) embeds into $\mathcal{T}\left(\Gamma_{m}\right) \simeq \mathcal{T}_{m+1}$, for every $m \geq 1$.

It follows from (34) that $\mathcal{T}_{0,5}$ is biholomorphic to $\mathcal{T}(\Gamma)$. By Royden's theorem, biholomorphic maps between Teichmüller spaces are necessarily isometries so there is a biholomorphic isometry between $\mathcal{T}_{0,5}$ and $\mathcal{T}(\Gamma)$. Combining this with the previous conclusion we find that $\mathcal{T}_{0,5}$ holomophically and isometrically embeds into each $\mathcal{T}_{g}, g \geq 2$. This completes the proof of the theorem.

\section{Appendix 2: Rigidity of translation inVARIANT FUnCTIONS INTO $\mathbb{H}$}

The goal of this appendix is to prove the following lemma that was used in Proposition 6.1. This result is a special case of Proposition 6.2 from the paper by Gekhtman [11]. The proof below closely follows Gekhtman's proof.

Lemma 11.1. Let $f: \mathbb{H}^{2} \rightarrow \mathbb{H}$ be a holomorphic function satisfying

$$
f(\lambda, \lambda)=\lambda,
$$

for all $\lambda \in \mathbb{H}$. If

$$
f\left(z_{1}+t, z_{2}+t\right)=f\left(z_{1}, z_{2}\right)+t,
$$

for all $\left(z_{1}, z_{2}\right) \in \mathbb{H}^{2}$ and all $t \in \mathbb{R}$, then

$$
f\left(z_{1}, z_{2}\right)=\alpha_{1} z_{1}+\alpha_{2} z_{2}
$$

for some $\alpha_{1}, \alpha_{2} \geq 0$

We start with the following proposition.

Proposition 11.1. Let $\phi: \mathbb{C} \rightarrow \mathbb{R}$ be a harmonic function with

$$
\phi(0)=\frac{\partial \phi}{\partial x}(0)=\frac{\partial \phi}{\partial y}(0)=0 .
$$

Suppose there is a $C>0$ so that $\phi(z) \geq-C|z|$ for all $z \in \mathbb{C}$. Then $\phi$ is identically zero.

Proof. We use the Poisson integral formula to show that $\phi$ has sublinear growth.

Write $\phi=\phi_{+}-\phi_{-}$, where $\phi_{+}(z)=\max \{0, \phi(z)\}$, and $\phi_{-}(z)=\max \{0,-\phi(z)\}$. Fix $r>0$, and set

$$
A=\int_{0}^{1} \phi_{+}\left(r e^{2 \pi i \theta}\right) d \theta, B=\int_{0}^{1} \phi_{-}\left(r e^{2 \pi i \theta}\right) d \theta .
$$


By the mean value property, $A-B=\phi(0)=0$. We compute

$$
\begin{aligned}
\int_{0}^{1}\left|\phi\left(r e^{2 \pi i \theta}\right)\right| d \theta & =A+B \\
& =2 B \\
& =2 \int_{0}^{1} \phi_{-}\left(r e^{2 \pi i \theta}\right) d \theta \\
& \leq 2 C r
\end{aligned}
$$

where in the last inequality, we've used $\phi(z) \geq-C|z|$. Now, for any $z \in \mathbb{D}_{r}(0)$, the Poisson integral formula for the disc $\mathbb{D}_{r}(0)$ yields

$|\phi(z)|=\left|\int_{0}^{1} \frac{r^{2}-\left(\frac{r}{2}\right)^{2}}{r \mid z-r e^{2 \pi i \theta \mid}} \phi\left(e^{2 \pi i \theta}\right) d \theta\right| \leq \sup _{\theta \in[0,2 \pi]}\left(\frac{3 r}{4 \mid z-r e^{2 \pi i \theta \mid}}\right) \int_{0}^{1}\left|\phi\left(r e^{2 \pi i \theta}\right)\right| d \theta$.

Considering only $z$ 's such that $|z|=\frac{r}{2}$, we have

$$
\sup _{\theta \in[0,2 \pi]}\left(\frac{3 r}{4\left|z-r e^{2 \pi i \theta}\right|}\right) \leq \frac{3}{2}
$$

and so derive the inequality

$$
|\phi(z)| \leq 3 C r=6 C|z|,
$$

for every $z \in \mathbb{C}$ such that $|z|=\frac{r}{2}$. By varying $r>0$ we derive the upper bound for every $z \in \mathbb{C}$.

Since $\phi$ is harmonic and has sublinear growth, $\phi$ is affine, that is, $\phi(x+i y)=$ $a x+b y+c$ for some $a, b, c \in \mathbb{C}$. (Indeed, the higher derivatives of $\phi$ at 0 vanish, as we can see by differentiating Poisson's formula on $B_{r}(0)$ under the integral and letting $r$ tend to infinity.) By assumption, $\phi$ and its first derivatives vanish at the origin, so $\phi$ is identically 0 .

11.1. Proof of Lemma 11.1. The idea is to first show that $f$ is of form

$$
f\left(z_{1}, z_{2}\right)=\sum_{j=1}^{2} \alpha_{j} z_{j}+H\left(z_{2}-z_{1}\right)
$$

for some entire function $H: \mathbb{C} \rightarrow \mathbb{C}$. Then we use Proposition 11.1 to show that $H \equiv 0$.

We begin with the proposition.

Proposition 11.2. There are $\alpha_{1}, \alpha_{2} \geq 0$ such that $\alpha_{1}+\alpha_{2}=1$ and

$$
\alpha_{j}=\frac{\partial f}{\partial z_{j}}(\lambda, \lambda)
$$

for $j=1,2$ and every $\lambda \in \mathbb{H}$.

Proof. Let $\iota(\lambda)=(\lambda, \lambda)$ be the diagonal embedding $\iota: \mathbb{H} \rightarrow \mathbb{H}^{2}$. From $f(\lambda, \lambda)=\lambda$ we find $f \circ \iota$ is the identity map on $\mathbb{H}$. Applying the chain rule we derive the identity

$$
\frac{\partial f}{\partial z_{1}}(\lambda, \lambda)+\frac{\partial f}{\partial z_{2}}(\lambda, \lambda)=1
$$

for every $\lambda \in \mathbb{H}$. 
On the other hand, we have the Schwarz lemma for the maps $h: \mathbb{H}^{2} \rightarrow \mathbb{H}$ (see Lemma 7.5.6 in [20] for the proof in the case of the polydisc)

$$
\operatorname{Im}\left(z_{1}\right)\left|\frac{\partial h}{\partial z_{1}}\left(z_{1}, z_{2}\right)\right|+\operatorname{Im}\left(z_{2}\right)\left|\frac{\partial h}{\partial z_{2}}\left(z_{1}, z_{2}\right)\right| \leq \operatorname{Im}\left(h\left(z_{1}, z_{2}\right)\right) .
$$

Replacing $\left(z_{1}, z_{2}\right)=(\lambda, \lambda)$ and $h=f$ (and again using $f(\lambda, \lambda)=\lambda$ ) we get

$$
\left|\frac{\partial f}{\partial z_{1}}\left(z_{1}, z_{2}\right)\right|+\left|\frac{\partial f}{\partial z_{2}}\left(z_{1}, z_{2}\right)\right| \leq 1
$$

Combining this with (38) shows that the holomorphic functions $\lambda \mapsto \frac{\partial f}{\partial z_{j}}(\lambda, \lambda)$ are non-negative, and so by the maximum principle they must be constant. Set

$$
\alpha_{j}=\frac{\partial f}{\partial z_{j}}(\lambda, \lambda) .
$$

The identity $\alpha_{1}+\alpha_{2}=1$ now follows from (38).

Let

$$
g\left(z_{1}, z_{2}\right)=f\left(z_{1}, z_{2}\right)-\sum_{j=1}^{2} \alpha_{j} z_{j},
$$

Then $g$ satisfies the conditions

$$
\begin{gathered}
g(\lambda, \lambda)=0, \\
\frac{\partial g}{\partial z_{j}}(\lambda, \lambda)=0,
\end{gathered}
$$

for every $\lambda \in \mathbb{H}$, and

$$
g\left(z_{1}+t, z_{2}+t\right)=g\left(z_{1}, z_{2}\right), \text { for all } t \in \mathbb{R} .
$$

Condition (41) implies that

$$
g\left(z_{1}+c, z_{2}+c\right)=g\left(z_{1}, z_{2}\right)
$$

for every $c \in \mathbb{C}$ such that $\operatorname{Im}(c) \geq 0$. Indeed, fixing $z_{1}, z_{2} \in \mathbb{H}$, the function $c \mapsto g\left(z_{1}+c, z_{2}+c\right)-g\left(z_{1}, z_{2}\right)$ is holomorphic in a neighborhood of $\overline{\mathbb{H}}$ and it vanishes on the real axis, thus, it vanishes on the whole domain.

Proposition 11.3. There exists an entire function $H: \mathbb{C} \rightarrow \mathbb{C}$ such that $H(0)=$ $H^{\prime}(0)=0$ and

$$
g\left(z_{1}, z_{2}\right)=H\left(z_{2}-z_{1}\right)
$$

for every $\left(z_{1}, z_{2}\right) \in \mathbb{H}^{2}$.

\section{Proof. Write}

where

$$
g\left(z_{1}, z_{2}\right)=h(\zeta, w)
$$

$$
\zeta=\sum_{j=1}^{2} \alpha_{j} z_{j} \text { and } w=z_{2}-z_{1},
$$

and $h$ is holomorphic on the image $\Omega$ of $\mathbb{H}$ under this (linear) coordinate change. For $\zeta \in \mathbb{H}$, let $\Omega(\zeta)$ be 


$$
\Omega(\zeta)=\{w \in \mathbb{C}:(\zeta, w) \in \Omega\}
$$

Then

$$
\Omega=\bigcup_{\zeta \in \mathbb{H}}(\zeta, \Omega(\zeta))
$$

Define $h^{\zeta}: \Omega(\zeta) \rightarrow \mathbb{C}$, the restriction of $g$ on the slice $\Omega(\zeta)$, by

$$
h^{\zeta}(w)=h(\zeta, w) .
$$

From (42) (and the fact $\alpha_{1}+\alpha_{2}=1$ ) we find that $h^{\zeta+c}(w)=h^{\zeta}(w)$, for every $\zeta \in \mathbb{H}, c \in \mathbb{C}$ with $\operatorname{Im}(c) \geq 0$, and $w \in \Omega^{\zeta} \cap \Omega^{\zeta+c}$. Therefore, it follows that for every $\zeta_{1}, \zeta_{2} \in \mathbb{H}$ we have

$$
h^{\zeta_{1}}(w)=h^{\zeta_{2}}(w), \text { for every } w \in \Omega^{\zeta_{1}} \cap \Omega^{\zeta_{2}}
$$

For each $\zeta \in \mathbb{H}, \Omega(\zeta)$ is a convex open set containing the origin. Moreover, $\Omega(t \zeta)=t \Omega(\zeta)$ for every $t>0$. It follows that $\Omega\left(i t_{1}\right) \subset \Omega\left(i t_{2}\right)$ for $0<t_{1}<t_{2}$, and that

$$
\bigcup_{t>0} \Omega(i t)=\mathbb{C} \text {. }
$$

Together with (43) this means that there is an entire function $H: \mathbb{C} \rightarrow \mathbb{C}$ so that

$$
h(\zeta, w)=h^{\zeta}(w)=H(w), \quad w \in \Omega(\zeta) .
$$

In particular, the function $h(\zeta, w)$ does not depend on $\zeta$.

We have shown that $h(\zeta, w)=H(w)$ for every $(\zeta, w) \in \Omega$ and the identity $g\left(z_{1}, z_{2}\right)=H\left(z_{2}-z_{1}\right)$ follows. The identity $H(0)=H^{\prime}(0)=0$ follows from the conditions (39), (40) and the chain rule.

The previous proposition together with the definition of $g\left(z_{1}, z_{2}\right)$ implies the idenity

$$
f\left(z_{1}, z_{2}\right)=\sum_{j=1}^{2} \alpha_{j} z_{j}+H\left(z_{2}-z_{1}\right),
$$

when $\left(z_{1}, z_{2}\right) \in \mathbb{H}$. (It is now clear that $f$ extends holomorphically to a function on $\mathbb{C}^{2}$ but we make no use of this fact). To complete the proof of the lemma, it thus suffices to show that $H$ is identically 0 .

Recall that $f\left(z_{1}, z_{2}\right) \in \mathbb{H}$ when $\left(z_{1}, z_{2}\right) \in \mathbb{H}^{2}$. Together with (44) this yields the inequality

$$
\operatorname{Im}\left(H\left(z_{2}-z_{1}\right)\right) \geq-\operatorname{Im}\left(\alpha_{1} z_{1}+\alpha_{2} z_{2}\right) .
$$

Let $w \in \mathbb{C}$ and $\epsilon>0$. If $\operatorname{Im}(w) \geq 0$ we let $z_{2}=w+\epsilon i$ and $z_{1}=\epsilon i$, and if $\operatorname{Im}(w) \leq 0$ we let $z_{2}=\epsilon i$ and $z_{1}=-w+\epsilon i$. Either way we have $\left(z_{1}, z_{2}\right) \in \mathbb{H}^{2}$ and $w=z_{2}-z_{1}$. From Proposition 11.2 we can conclude

$$
\operatorname{Im}\left(\alpha_{1} z_{1}+\alpha_{2} z_{2}\right) \leq \max \left\{\left|z_{1}\right|,\left|z_{2}\right|\right\} \leq|w|+2 \epsilon .
$$

Together with (45) we verify that the inequality

$$
\operatorname{Im}(H(w)) \geq-(|w|+2 \epsilon),
$$


holds for every $w \in \mathbb{C}$ and every $\epsilon>0$. Letting $\epsilon \rightarrow 0$ grants us the inequality

$$
\operatorname{Im}(H(w)) \geq-|w| \text {. }
$$

Since by Proposition 11.3 we have $H(0)=H^{\prime}(0)=0$, after applying Proposition 11.1 to the harmonic function $\operatorname{Im}(H)$ we conclude $H \equiv 0$. The proof of the lemma is complete.

\section{REFERENCES}

[1] J. Agler, N. Young, The hyperbolic geometry of the symmetrized bidisc. Journal Geom. Anal. 14 , no. 3, 375-403 (2004)

[2] L. Bers, Fiber spaces over Teichmüller spaces. Acta Math. 130 (1973)

[3] C. Costara, The symmetrized bidisc and Lempert's theorem. Bulletin London Math. Soc. 36, no. 5, 656-662 (2004)

[4] J.P. D'Angelo, Several Complex Variables and the Geometry of Real Hypersurfaces. CRC, Boca Raton, FL, (1993)

[5] C. Earle, On the Carathèodory metric in Teichmüller spaces. Discontinuous groups and Riemann surfaces, 99-103. Ann. of Math. Studies, No. 79, Princeton Univ. Press, Princeton, N.J. (1974)

[6] C. Earle, I. Kra, On holomorphic mappings between Teichmüller spaces. Contributions to analysis (a collection of papers dedicated to Lipman Bers), pp. 107-124. Academic Press, New York, (1974)

[7] C. Earle, L. Harris, J. Hubbard, S. Mitra, Sudeb, Schwarz's lemma and the Kobayashi and Carathéodory pseudometrics on complex Banach manifolds. Kleinian groups and hyperbolic 3-manifolds (Warwick, 2001), 363-384, London Math. Soc. Lecture Note Ser., 299, Cambridge Univ. Press, Cambridge, (2003)

[8] A. Edmonds, J. Ewing, R. Kulkarni, Torsion free subgroups of Fuchsian groups and tessellations of surfaces. Inventiones Math. 69, 331-346 (1982)

[9] F. Gardiner, Approximation of infinite-dimensional Teichmüller spaces. Trans. Amer. Math. Soc. 282 no. 1, 367383.(1984)

[10] F. Gardiner, Caratheodory's and Kobayashi metrics on Teichmüller space, draft

[11] D. Gekhtman, Asymptotics of the translation flow on holomorphic maps out of the poly-plane. preprint arXiv: 1702.02177

[12] J. Hubbard, H. Masur, Quadratic differentials and foliations. Acta Math. 142 no. 3-4, 221-274. (1979)

[13] J. Jenkins, On the Existence of Certain General Extremal Metrics. Annals of Mathematics, Vol. 66, No. 3 , pp. 440-453, (1957)

[14] G. Knese, A Schwarz lemma on the polydisk. Proc. Amer. Math. Soc. 135 no. 9, 2759-2768 (2007)

[15] I. Kra, The Carathèodory metric on abelian Teichmüller disks. Journal Analyse Math. 40 (1981), 129-143 (1982)

[16] L. Lempert, The Kobayashi metric and the representation of domains on the ball. Bull. Soc. Math. France 109 , no. 4, 427-474 (1981)

[17] J. Liu, On the existence of Jenkins-Strebel differentials. Bull. London Math. Soc. 36 no. 3, 365-377 (2004)

[18] C. McMullen, Billiards and Teichmller curves on Hilbert modular surfaces. Journal Amer. Math. Soc. 16 , no. 4, 857-885 (2003)

[19] H. Royden, Automorphisms and isometries of Teichmüller space. Advances in the Theory of Riemann Surfaces (Proc. Conf., Stony Brook, N.Y., 1969) pp. 369-383 Ann. of Math. Studies, No. 66. Princeton Univ. Press, Princeton, N.J. (1971)

[20] W. Rudin, Function Theory in Polydiscs W. A. Benjamin, Inc., New York-Amsterdam (1969)

Department of Mathematics

CAlifornia Institute of Technology

Pasadena, CA 91125, USA

E-mail address: markovic@caltech.edu 